\title{
Numerical study and stability of the Lengyel-Epstein chemical model with diffusion
}

\author{
Zain UI Abadin Zafar ${ }^{1}$, Zahir Shah ${ }^{2 *}$ (D) Nigar Ali ${ }^{3}$, Poom Kumam ${ }^{4,5^{*}}$ (D) and Ebraheem O. Alzahrani ${ }^{6}$
}

\author{
"Correspondence: \\ zahir.sha@kmutt.ac.th; \\ poom.kum@kmutt.ac.th \\ ${ }^{2}$ Center of Excellence in Theoretical \\ and Computational Science \\ (TaCS-CoE), SCL 802 Fixed Point \\ Laboratory, Science Laboratory \\ Building, King Mongkut's University \\ of Technology Thonburi (KMUTT), \\ 126 Pracha-Uthit Road, Bang Mod, \\ Thrung Khru, Bangkok 10140, \\ Thailand \\ ${ }^{4}$ KMUTT Fixed Point Research \\ Laboratory, Room SCL 802 Fixed \\ Point Laboratory, Science \\ Laboratory Building, Department of \\ Mathematics, Faculty of Science, \\ King Mongkut's University of \\ Technology Thonburi (KMUTT), 126 \\ Pracha-Uthit Road, Bang Mod, \\ Thrung Khru, Bangkok 10140, \\ Thailand \\ Full list of author information is \\ available at the end of the article
}

\begin{abstract}
In this paper, a nonlinear mathematical model with diffusion is taken into account to review the dynamics of Lengyel-Epstein chemical reaction model to describe the oscillating chemical reactions. For this purpose, the dimensionless Lengyel-Epstein model with diffusion and homogeneous boundary condition is considered. The steady states with and without diffusion of the Lengyel-Epstein model are studied. The basic reproductive number is computed and the global steady states for the system are calculated. Numerical results are offered for two systems using three well known techniques to validate the main outcomes. The consequences established from this qualitative study are supported by numerical simulations characterized by distinct programs, adopting forward Euler method, Crank-Nicolson method, and nonstandard finite difference method.
\end{abstract}

Keywords: Lengyel-Epstein chemical reaction (LECR) model; Mathematical modeling; Forward Euler method; Stability analysis; Crank-Nicolson method; Equilibrium nodes; Nonstandard finite difference method 
(b) The iodide ion oxidation by free chlorine dioxide radical is given in the second equation of (1).

(c) A reaction of iodide and chlorite ions created in the first and second equation of (1) generates iodine as given in the third equation of (1).

The rate equations for the so-called $\mathrm{ClO}_{2}-I_{2}-\mathrm{MA}$ (chlorine dioxide-iodine-malonic acid) are given by

$$
\begin{aligned}
& -\frac{d\left[I_{2}\right]}{d \tau}=\frac{k_{1}[\mathrm{MA}]\left[I_{2}\right]}{k_{2}+\left[I_{2}\right]}, \\
& -\frac{d\left[\mathrm{ClO}_{2}\right]}{d \tau}=k_{3}\left[\mathrm{ClO}_{2}\right]\left[I^{-}\right]
\end{aligned}
$$

and

$$
-\frac{d\left[\mathrm{ClO}_{2}^{-}\right]}{d \tau}=k_{4}\left[\mathrm{H}^{+}\right]\left[\mathrm{ClO}_{2}^{-}\right]\left[I^{-}\right]+\frac{k_{5} I^{-}}{\alpha+\left[I^{-}\right]^{2}}\left[I^{-}\right]\left[I_{2}\right]\left[\mathrm{ClO}_{2}^{-}\right]
$$

where $k_{1}, k_{3}, k_{4}$, and $k_{5}$ are reaction rate constants, whereas $k_{2}$ and $\alpha$ denote saturation levels. Furthermore, the last term in (iii) characterizes the autocatalytic consequence of $I_{2}$ and the self-inhibitory outcome of $I^{-}$on the chlorite-iodide reaction [2]. This framed term disappears when $\left[I^{-}\right] \rightarrow 0$, where no reaction can happen since no iodide is accessible, and in the limit $\left[I^{-}\right] \rightarrow \infty$, where the intense self-inhibition develops. The aforementioned rate equations (i)-(iii) form a five-variable system containing $\left[\mathrm{ClO}_{2}^{-}\right],\left[\mathrm{I}^{-}\right],\left[\mathrm{ClO}_{2}\right],\left[\mathrm{I}_{2}\right]$, and $[M A]$. Nonetheless, the iodization of Malonic acid helps largely as a cradle of iodine ions, and $M A$ can be swapped by ethyl acetoacetate [2]. Furthermore, it is experimentally seen that the concentrations of chlorite and iodide ions fluctuate over numerous orders of level through an oscillation, whereas the concentrations of chlorine dioxide and malonic acid vary slowly. These concentrations may consequently be considered as constants, and actions of the system may be estimated by a two-variable specimen including only the concentrations of iodide and chlorine ions. For a flow reactor with appropriate serving, it is conceivable to retain the concentrations of malonic acid, chlorine dioxide and iodine approximately constant, and oscillations can still be perceived in the suitable ranges of temperature and concentrations. Thus, we conclude that $\mathrm{MA}, \mathrm{ClO}_{2}$ and $I_{2}$ vary much more sluggishly than the intermediate $\mathrm{ClO}_{2}{ }^{-}$and $\mathrm{I}^{-}$which change by some orders of level during an oscillation period. Taking $U=I^{-}, V=\mathrm{ClO}_{2}{ }^{-}$and $\mathrm{H}=\mathrm{I}_{2}$, we obtain the following equations $[3,4]$ :

$$
\begin{array}{ll}
H \rightarrow U, & z_{11}=k_{1}^{\prime},
\end{array}
$$

and

$$
4 U+V \rightarrow Q, \quad z_{33}=\frac{k_{2}^{\prime}[U][V]}{\alpha+[U]^{2}}, \quad k_{3}^{\prime}=k_{5}\left[I_{2}\right]
$$

Arguing as in [3], an improved application in scientific modeling, it is vital that models are to be written dimensionless. This state is achieved if we make the transforma- 
tions

$$
U=\sqrt{\alpha} u, \quad V=\frac{\alpha k_{2}^{\prime}}{k_{3}^{\prime}} \nu, \quad \tau=\frac{t}{k_{2}^{\prime}}, \quad l=\frac{k_{1}^{\prime}}{\sqrt{\alpha} k_{2}^{\prime}} \quad \text { and } \quad m=\frac{k_{3}^{\prime}}{\sqrt{\alpha} k_{2}^{\prime}} .
$$

Under these transformations, we have the following Lengyel-Epstein model [5]:

$$
\left\{\begin{array}{l}
\frac{d u}{d t}=-u+l-4 \psi(u) v:=G(v, u) \\
\frac{d v}{d t}=m u-m \psi(u) v:=H(v, u)
\end{array}\right.
$$

Kinetic applications, in elucidation, are a controlling gizmo in the study of the reaction structure, allowing to gather vital facts of the processes that transpire before the influential phase of the speed. Through kinetic investigation one can decide the speed law of a reaction just as its steady rate. One of the methodologies for this is the utilization of integrated equations. In this methodology, one checks whether the adjustment in the concentration of one of the reactants or items follows first or second order kinetics or, all the more once in a while, kinetics with higher orders or even zero order. In a reaction, reactants go through a progress state zone along the reaction organize among products and reactants, where chemical bonds are broken and changed. This change state was first projected by Eyring and Polanyi in the mid-1930s. Numerous scientists controlled pretty much every part of a chemical reaction, and have assumed a focal visionary aspect in the advancement of chemistry as a part of science. Along these lines, watching and understanding the progress state have been viewed as the "Holy Grail" of chemistry [6]. Model (6) is a nonfractional order system, i.e., it contains the first order derivative with respect to time variable $\tau$. The first-order derivative with respect to the variable $\tau$ infers the transient change pace of these chemical reactions. Though, as a result of the intricacy of reactions which were biochemical in nature, chemical reaction practices are regularly influenced by or rely on the historical background of chemical reactions. Many researchers have solved the Lengyel-Epstein chemical model [7-10]. A number of researchers were using fractional order techniques and they claimed these to be more suitable than classical ones [11-26].

In this paper, we are keen on an old style version of the Lengyel-Epstein reaction diffusion structure as a specimen of the chlorite-iodide-malonic-acid (CIMA) reaction. The deliberated model has been pulled into the light of legitimate concern for some analysts since its origin in 1991. The purpose behind this intrigue is the way that CIMA reaction is perhaps the most punctual trial that restricted the theoretical suggestions of Alan Turing in 1952, concerning the chemical basis for morphogenesis and, all the more by and large, pattern formation [27].

This paper is systematized into five sections. The introduction is the first section in which we intricate some history of the Lengyel-Epstein model in kinetic studies. In Sect. 2, we will study the Lengyel-Epstein model with diffusion. Besides this, we will study the steady states with and without diffusion of the Lengyel-Epstein model. In Sect. 3, we ponder the global steady states for the system. In Sects. 4 and 5, numerical finding are offered for two systems using three well known techniques to validate the main outcomes. Results and Discussion are presented in Sect. 6, and conclusions are drawn in Sect. 7. 


\section{Model with diffusion}

Here, we consider one-dimensional coupled Lengyel-Epstein model:

$$
\left\{\begin{array}{l}
\frac{d u}{d t}=\kappa_{1} \Delta u-u+l-4 v \psi(u), \quad \tau>0, x \in \Upsilon, \\
\frac{d v}{d t}=\kappa_{2} \Delta v+m u-m v \psi(u), \quad \tau>0, x \in \Upsilon, \\
u(x, 0)=u_{0} ; \quad v(x, 0)=v_{0}, \quad x \in \Upsilon, \\
u_{x}(x, \tau)=v_{x}(x, \tau)=0, \quad x \in \partial \Upsilon, \tau>0,
\end{array}\right.
$$

where $\Upsilon$ is a bounded domain in $\mathbb{R}^{N}$ with sufficiently smooth boundary $\partial \Upsilon$. Here, $u=$ $u(x, \tau)$ and $v=v(x, \tau)$ denote the concentration of the inhibitor chlorite $\left(\mathrm{ClO}_{2}\right)$ and the activator iodide $\left(I^{-}\right)$, respectively, at time $\tau>0$ and point $x \in \Upsilon$. The constants $l$ and $m$ are restrictions depending on the concentration of the starch, broadening the diffusion ratio to be effective by $\kappa_{1}$ and $\kappa_{2}$. The constants $\kappa_{1}, \kappa_{2}, l$ and $m$ are nonnegative. The function $\psi$ is supposed to be positive and continuously differentiable on $\mathbb{R}^{+}$such that $\psi(0)=0$, and for $u \in(0, l)$,

$$
\begin{aligned}
& \psi(l)>0, \\
& \psi(u) \geq \psi^{\prime}(u) u .
\end{aligned}
$$

\subsection{Invariant regions}

Now, we study the invariant zones for the system (7).

Definition $1([28,29])$ A frame $\mathfrak{A}=\left(0, \mathfrak{p}_{1}\right) \times\left(0, \mathfrak{p}_{2}\right)$ is called invariant if the vector field $(G, H)$ on the boundary $\partial \mathfrak{A}$ points inside, i.e.,

$$
\left\{\begin{array}{lll}
0 \leq G(0, v) \quad \text { and } \quad 0 \geq G\left(\mathfrak{p}_{1}, v\right) & \text { for } \mathfrak{p}_{2}>v>0 \\
0 \leq H(u, 0) & \text { and } \quad 0 \geq G\left(\mathfrak{u}, \mathfrak{p}_{2}\right) & \text { for } \mathfrak{p}_{1}>v>0 .
\end{array}\right.
$$

Proposition 1 The structure (7) satisfying condition (8) defines the invariant zone

$$
\mathfrak{A}_{\mathfrak{l}}=(a, l) \times\left(a, \frac{l}{\psi(l)}\right), \quad a=0 .
$$

\subsection{Steady states of model (6) without diffusion}

We shall consider system (6) without diffusion.

Proposition 2 The structure (6) has the unique steady state solution

$$
\left(u^{*}, v^{*}\right)=\left(\mu, \frac{\mu}{\psi(\mu)}\right), \quad \text { where } \mu=\frac{l}{5} .
$$

If the inequality $-\left[\psi(\mu)+4 \mu \psi^{\prime}(\mu)\right]<m \psi^{2}(\mu)$ is maintained, the steady state is an asymptotically stable equilibrium for the structure (6).

Proof An equilibrium node $\left(u^{*}, v^{*}\right)$ of (6) solves the system

$$
\left\{\begin{array}{l}
-u+l-4 v \psi(u)=G(u, v):=0 \\
m u-m v \psi(u)=H(u, v):=0
\end{array}\right.
$$


Simple calculations from (12) provide the solution given below:

$$
E_{0}\left(u^{*}, v^{*}\right)=\left(\mu, \frac{\mu}{\psi(\mu)}\right), \quad \text { where } \mu=\frac{l}{5} .
$$

Next, we ponder the stability of the equilibrium. The Jacobian matrix for the structure (6) at the equilibrium point $\left(u^{*}, v^{*}\right)$ is given by

$$
J\left(E_{0}\right)=\left(\begin{array}{cc}
-1-\frac{4 \mu}{\psi(\mu)} \psi^{\prime}(\mu) & -4 \psi(\mu) \\
m-\frac{m \mu}{\psi(\mu)} \psi^{\prime}(\mu) & -m \psi(\mu)
\end{array}\right) .
$$

We have

$$
J\left(E_{0}\right)=5 m \psi(\mu)=\frac{5 m \mu}{1+\mu^{2}}>0,
$$

and

$$
\operatorname{tr} J\left(E_{0}\right)=\left(-1-\frac{4 \mu}{\psi(\mu)} \psi^{\prime}(\mu)-m \psi(\mu)\right)=\frac{3 \mu^{2}-5-m \mu}{1+\mu^{2}}<0 .
$$

Thus, the Jacobian of (14) has eigenvalues with negative real parts. Hence, the equilibrium node of (14) is asymptotically stable, which is bound by condition (13). Hence, the proof of Proposition 2 is completed.

Comment 1 Observe that $0>G_{u}\left(u^{*}, v^{*}\right), 0>H_{v}\left(u^{*}, v^{*}\right)$ and $0>H_{u}\left(u^{*}, v^{*}\right)$. If

$$
-1-\frac{4 \mu}{\psi(\mu)} \psi^{\prime}(\mu)=F_{u}\left(u^{*}, v^{*}\right)>0
$$

is satisfied then we call $u(x, t)$ an activator, $v(x, t)$ an inhibitor, and the structure (6) is an activator-inhibitor structure. Since $\psi(\mu)$ is always positive, multiplying both sides of the inequity by $\psi(\mu)$, we get

$$
-\left[\psi(\mu)+4 \mu \psi^{\prime}(\mu)\right]>0 .
$$

Comment 2 Merging the activator-inhibitor condition (18) with the steady state condition given in Proposition 2, we discover that the condition

$$
m \psi^{2}(\mu)>-\left[\psi(\mu)+4 \mu \psi^{\prime}(\mu)\right]>0
$$

makes the system (6) a diffusion free stable activator-inhibitor structure.

\subsection{Steady states of model (6) with diffusion}

In this subsection, we shall debate the basic properties of the nonhomogeneous steady states of the Lengyel-Epstein structure. The steady state points satisfy the following system:

$$
\left\{\begin{array}{l}
0=\kappa_{1} \Delta u-u+l-4 \psi(u) v \\
0=\kappa_{2} \Delta v+m(u-\psi(u) v)
\end{array}\right.
$$

with the homogeneous Neumann boundary conditions $u_{x}(x, \tau)=v_{x}(x, \tau)=0, x \in \partial \Upsilon$. 
Definition 2 A constant solution is supposed to be Turing unstable if it is stable in the absence of diffusion, whereas it transforms into unstable one when diffusion is available.

Proposition 3 ([30]) Assume that $g \in C(\bar{\Upsilon} \times \mathbb{R})$ and $w \in C^{2}(\Upsilon) \cap C^{1}(\bar{\Upsilon})$. It follows that:

(a) If $0 \leq \Delta w(x)+g(x, w(x))$ in $\Upsilon$, with $0 \geq w_{x}$ on $\partial \Upsilon$ and $\max _{\bar{\gamma}}(w(x))=w\left(x_{0}\right)$, then

$$
0 \leq g\left(x_{0}, w\left(x_{0}\right)\right)
$$

(b) If $0 \geq \Delta w(x)+g(x, w(x))$ in $\Upsilon$, with $0 \leq w_{x}$ on $\partial \Upsilon$ and $\min _{\bar{\Upsilon}}(w(x))=w\left(x_{0}\right)$, then

$$
0 \geq g\left(x_{0}, w\left(x_{0}\right)\right)
$$

Lemma 1 Conditions (10) and (11) imply

$$
\psi^{\prime}(0) \geq \frac{\psi(u)}{u}>0
$$

Proof Let $u \psi^{\prime}(u) \leq \psi(u)$, which is equivalent to

$$
\left(\frac{\psi(u)}{u}\right)^{\prime}=\frac{-\psi(u)+u \psi^{\prime}(u)}{u^{2}} \leq 0 .
$$

Hence, $\frac{\psi(u)}{u}$ is a decreasing function. Now, for some $s \in(0, u)$, we have

$$
\frac{\psi(u)}{u} \leq \frac{\psi(s)}{s} \Rightarrow \lim _{s \rightarrow 0} \frac{\psi(s)}{s} \geq u^{-1} \psi(u)
$$

This yields

$$
\frac{\psi(u)}{u} \leq \psi^{\prime}(0)
$$

Proposition 4 If $(u, v)=(u(x), v(x))$ is a positive equilibrium of the boundary value problem (19), then $\varepsilon_{2}<u<\varepsilon_{1}$ and $\frac{\varepsilon_{2}}{\psi\left(\varepsilon_{2}\right)}<v<\frac{\varepsilon_{1}}{\psi\left(\varepsilon_{1}\right)}$, for all $x \in \Upsilon$, where $\varepsilon_{1}=l$, $\varepsilon_{2}=\frac{\varepsilon_{1}}{1+4 \psi^{\prime}(0) \frac{\varepsilon_{1}}{\psi\left(\varepsilon_{1}\right)}}$.

Proof If at some node in $\bar{\Upsilon}$ the function $u$ reaches its extreme over $\bar{\Upsilon}$, then by (19) and Proposition 3, at this point we have

$$
l-u-4 v \psi(u) \geq 0 .
$$

It implies that $u<l$. Likewise, if $v$ achieves an extreme over $\bar{\Upsilon}$ at some node, then by (19) and Proposition 3, we have $0 \leq u-4 v \psi(u)$, inferring that $v \leq \frac{u}{4 \psi(u)} \leq \frac{\mu}{\psi(\mu)}$. Since (11) warranties that $u(\psi(u))^{-1}$ is growing for $u$ between 0 and $\varepsilon_{1}$. If $u$ achieves its minimum over $\bar{\Upsilon}$ at some point, then

$$
l \leq 4 v \psi(u)+u \leq u+4 u \frac{\psi(u)}{u}\left(\frac{\varepsilon_{1}}{\psi\left(\varepsilon_{1}\right)}\right) .
$$

This, along with condition (20), yields

$$
u\left[1+4\left(\frac{\psi(u) \varepsilon_{1}}{u \psi\left(\varepsilon_{1}\right)}\right)\right]>l .
$$


Therefore, $\varepsilon_{2}<u$. Also, if $v$ has a minimum over $\bar{\Upsilon}$ at some point, then $v \psi(u) \geq u$. Therefore, we get $\frac{u}{\psi(u)} \leq v$ which leads to $\frac{\varepsilon_{2}}{\psi\left(\varepsilon_{2}\right)} \leq v$.

\section{Global asymptotic stability}

In this section, we study the global asymptotic stability of the system (7). The reason is to find some adequate conditions for the global stability of the steady state equations. First, assume that $h_{a}(u)=(\psi(u))^{-1}(l-u)$, which results in $h_{a}\left(u^{*}\right)=\mu(\psi(u))^{-1}$. Now, system (7) can be rewritten as

$$
\left\{\begin{array}{l}
u_{\tau}=\kappa_{1} \Delta u+\left[-4\left(v-v^{*}\right)+\left(h_{a}(u)-h_{a}\left(u^{*}\right)\right)\right] \psi(u), \\
v_{\tau}=\kappa_{2} \Delta v+m\left[-\left(v-v^{*}\right)+\left(\frac{u}{\psi(u)}-\frac{u^{*}}{\psi\left(u^{*}\right)}\right)\right] \psi(u) .
\end{array}\right.
$$

Theorem 1 Assume that condition (11) is satisfied and $\frac{\psi^{\prime}(u)}{\psi(u)} \geq-\frac{1}{l-u}$. It follows that for any solution $(u, v)$ to $(7)$, we get

$$
\lim _{\tau \rightarrow 0}\left\|v(\cdot, \tau)-u v^{*}\right\|_{L^{2}(\Upsilon)}=\lim _{\tau \rightarrow 0}\left\|u(\cdot, \tau)-u^{*}\right\|_{L^{2}(\Upsilon)}=0 .
$$

Lemma 2 ([7]) If $u \in(0, l)$, then there exist a real $\theta$ between $\mu$ and $u$ and a nonnegative function $\chi(\theta)$, which is continuous, such that

$$
\frac{u}{\psi(u)}-\frac{\mu}{\psi(\mu)}=\mu(u-\mu) \chi(\theta)
$$

Proof Letting $s=\frac{u}{\mu}$ and $w(s)=\frac{s \mu}{\psi(\mu s)}$, we have

$$
\frac{u}{\psi(u)}-\frac{\mu}{\psi(\mu)}=w(s)-w(1)
$$

Using the mean value theorem, for suitable $\delta_{1}$ we have

$$
w(s)-w(1)=(s-1) w^{\prime}\left(\delta_{1}\right)
$$

Also

$$
w^{\prime}(s)=\frac{d}{d s}\left(\frac{s \mu}{\psi(\mu s)}\right)=\mu \frac{-u \psi^{\prime}(u)+\psi(u)}{\psi^{2}(u)},
$$

where $\mu s=u$. Now, suppose that $\chi(u)=\frac{\psi(u)-u \psi^{\prime}(u)}{\psi^{2}(u)}$, then (30) reads $w^{\prime}\left(\delta_{1}\right)=\mu \chi(\theta)$, which guarantees that $\chi(\theta) \geq 0$.

\section{Numerical schemes}

In this section, we look at two specific examples and use numerical analysis based on forward Euler, Crank-Nicolson, and nonstandard methods to investigate the solution of the system and its stability. 


\subsection{The CIMA model}

In this first example, we suppose $\psi(u)=u\left(1+u^{2}\right)^{-1}$, then the system (7) takes the form

$$
\left\{\begin{array}{l}
u_{\tau}=l-u-4 \frac{u}{1+u^{2}} v+\kappa_{1} \Delta u \\
v_{\tau}=m u-\frac{m u v}{1+u^{2}}+\kappa_{2} \Delta v
\end{array}\right.
$$

To discretize system (20) using the finite difference method, firstly $[0, \Im]^{2} \times[0, \mathrm{~T}]$ is partitioned into $M^{2} \times N$ parts with spatial and temporal step sizes $d x=\frac{\Im}{M}$ and $d \tau=\frac{T}{N}$. Then, the grid points are $x_{i}=i d x$ and $\tau_{j}=j d \tau$, where $i=0,1,2, \ldots, M$ and $j=0,1,2, \ldots, N$. We represent $u_{i}^{j}$ and $v_{i}^{j}$ as the finite difference approximations of $u(i d x, j d \tau)$ and $v(i d x, j d \tau)$, respectively. First order temporal derivative and second order spatial derivative finite difference formulas are:

$$
\begin{aligned}
& \left.u_{\tau}\right|_{i} ^{j}=\delta_{\tau} u_{i}^{j}=\frac{u_{i}^{j+1}-u_{i}^{j}}{d \tau},\left.\quad v_{\tau}\right|_{i} ^{j}=\delta_{\tau} v_{i}^{j}=\frac{v_{i}^{j+1}-v_{i}^{j}}{d \tau}, \\
& \left.u_{x x}\right|_{i} ^{j}=\delta_{x}^{2} u_{i}^{j}=\frac{u_{i-1}^{j}-2 u_{i}^{j}+u_{i+1}^{j}}{(d x)^{2}},\left.\quad v_{x x}\right|_{i} ^{j}=\delta_{x}^{2} v_{i}^{j}=\frac{v_{i-1}^{j}-2 v_{i}^{j}+v_{i+1}^{j}}{(d x)^{2}} .
\end{aligned}
$$

\subsection{Forward Euler method}

Substituting the values of $\left.u_{\tau}\right|_{i} ^{j}$ and $\left.u_{x x}\right|_{i} ^{j}$ into the first equation of (20) and the values of $\left.v_{\tau}\right|_{i} ^{j}$ and $\left.v_{x x}\right|_{i} ^{j}$ into the second equation of (20), we have

$$
\delta_{\tau} u_{i}^{j}=\kappa_{1} \delta_{x}^{2} u_{i}^{j}+l-u_{i}^{j}-4 \frac{u_{i}^{j} v_{i}^{j}}{1+\left(u_{i}^{j}\right)^{2}}, \quad \delta_{\tau} v_{i}^{j}=\kappa_{2} \delta_{x}^{2} v_{i}^{j}+m\left(u_{i}^{j}-\frac{u_{i}^{j} v_{i}^{j}}{1+\left(u_{i}^{j}\right)^{2}}\right) .
$$

After simple calculation, we have

$$
\begin{aligned}
& u_{i}^{j+1}=R_{1}\left(u_{i-1}^{j}-2 u_{i}^{j}+u_{i+1}^{j}\right)+u_{i}^{j}+l d \tau-u_{i}^{j} d \tau-\frac{4 u_{i}^{j} v_{i}^{j} d \tau}{1+\left(u_{i}^{j}\right)^{2}}, \\
& v_{i}^{j+1}=+R_{2}\left(v_{i-1}^{j}-2 v_{i}^{j}+v_{i+1}^{j}\right)+v_{i}^{j}+m u_{i}^{j} d \tau-\frac{m u_{i}^{j} v_{i}^{j} d \tau}{1+\left(u_{i}^{j}\right)^{2}},
\end{aligned}
$$

where $R_{1}=\frac{\kappa_{1} d \tau}{(d x)^{2}}$ and $R_{2}=\frac{\kappa_{2} d \tau}{(d x)^{2}}$. This technique is conditionally stable for $\frac{2-d \tau}{4}>R_{1}$ and $\frac{1}{2}>R_{2}$ for the structure (20).

\subsection{Crank-Nicolson method}

Substituting the values of $\left.u_{\tau}\right|_{i} ^{j},\left.u_{x x}\right|_{i} ^{j}$ and $\left.u_{x x}\right|_{i} ^{j+1}$ into the first equation of (32) and the values of $\left.v_{\tau}\right|_{i} ^{j},\left.v_{x x}\right|_{i} ^{j}$ and $\left.v_{x x}\right|_{i} ^{j+1}$ into the second equation of (32), we have

$$
\begin{aligned}
& \delta_{\tau} u_{i}^{j}=\frac{\kappa_{1}}{2} \delta_{x}^{2}\left(u_{i}^{j}+u_{i}^{j+1}\right)+l-u_{i}^{j}-4 \frac{u_{i}^{j} v_{i}^{j}}{1+\left(u_{i}^{j}\right)^{2}}, \\
& \delta_{\tau} v_{i}^{j}=\frac{\kappa_{2}}{2} \delta_{x}^{2}\left(v_{i}^{j}+v_{i}^{j+1}\right)+m\left(u_{i}^{j}-\frac{u_{i}^{j} v_{i}^{j}}{1+\left(u_{i}^{j}\right)^{2}}\right) .
\end{aligned}
$$


After simple calculation, we have the following scheme for system (32):

$$
\begin{aligned}
& u_{i}^{j+1}-\frac{R_{1}}{2}\left(u_{i+1}^{j+1}-2 u_{i}^{j+1}+u_{i-1}^{j+1}\right)=\frac{R_{1}}{2}\left(u_{i-1}^{j}-2 u_{i}^{j}+u_{i+1}^{j}\right)+u_{i}^{j}+l d \tau-u_{i}^{j} d \tau-\frac{4 u_{i}^{j} v_{i}^{j} d \tau}{1+\left(u_{i}^{j}\right)^{2}}, \\
& v_{i}^{j+1}-\frac{R_{2}}{2}\left(v_{i+1}^{j+1}-2 v_{i}^{j+1}+v_{i-1}^{j+1}\right)=\frac{R_{2}}{2}\left(v_{i-1}^{j}-2 v_{i}^{j}+v_{i+1}^{j}\right)+v_{i}^{j}+m u_{i}^{j} d \tau-\frac{m u_{i}^{j} v_{i}^{j} d \tau}{1+\left(u_{i}^{j}\right)^{2}},
\end{aligned}
$$

where $R_{1}=\frac{\kappa_{1} d \tau}{(d x)^{2}}$ and $R_{2}=\frac{\kappa_{2} d \tau}{(d x)^{2}}$. This scheme is unconditionally stable for the system (32).

\subsection{Nonstandard finite difference method}

Here, we will design a nonstandard finite difference method for the system (20). Substituting the values of $\left.u_{\tau}\right|_{i} ^{j},\left.u_{x x}\right|_{i} ^{j},\left.v_{\tau}\right|_{i} ^{j}$ and $\left.v_{x x}\right|_{i} ^{j}$ into equation (20), we have

$$
\begin{aligned}
& \frac{u_{i}^{j+1}-u_{i}^{j}}{d \tau}=\frac{\kappa_{1}}{(d x)^{2}}\left(u_{i-1}^{j}-2 u_{i}^{j}+u_{i+1}^{j}\right)+l-u_{i}^{j}-\frac{4 u_{i}^{j} v_{i}^{j}}{1+\left(u_{i}^{j}\right)^{2}}, \\
& \frac{v_{i}^{j+1}-v_{i}^{j}}{d \tau}=\frac{\kappa_{2}}{(d x)^{2}}\left(v_{i-1}^{j}-2 v_{i}^{j}+v_{i+1}^{j}\right)+m u_{i}^{j}-\frac{m u_{i}^{j} v_{i}^{j}}{1+\left(u_{i}^{j}\right)^{2}} .
\end{aligned}
$$

After simple calculation, we obtain the following explicit nonstandard finite difference scheme:

$$
\left\{\begin{array}{l}
u_{i}^{j+1}=\frac{R_{1}\left(u_{i-1}^{j}+u_{i+1}^{j}\right)+u_{i}^{j}+l d \tau}{\left(\frac{4 v_{i}^{j} d \tau}{1+\left(u_{i}^{j}\right)^{2}}+1+2 R_{1}+d \tau\right)}, \\
v_{i}^{j+1}=\frac{R_{2}\left(v_{i-1}^{j}+v_{i+1}^{j}\right)+v_{i}^{j}+m u_{i}^{j} d \tau}{\left(1+2 R_{2}+\frac{m u_{i}^{j} d \tau}{1+\left(u_{i}^{j}\right)^{2}}\right)},
\end{array}\right.
$$

where $R_{1}=\frac{\kappa_{1} d \tau}{(d x)^{2}}$ and $R_{2}=\frac{\kappa_{2} d \tau}{(d x)^{2}}$. This technique is completely stable for the structure (32).

\subsection{Stability analysis of nonstandard finite difference method}

To find the stability bounds, the von Neumann stability technique is used. After linearizing equation (33) and then using the von Neumann stability bounds, we get

$$
\begin{aligned}
& T(t+\Delta t) e^{i \alpha x}=e^{i \alpha x} T(t)+R_{1}\left(-2 T(t+\Delta t) e^{i \alpha x}+T(t) e^{i \alpha(x-\Delta x)}+T(t) e^{i \alpha(x+\Delta x)}\right) \\
& -d \tau e^{i \alpha x} T(t+\Delta t) \\
& \frac{T(t+\Delta t)}{T(t)}=\frac{\left(2 R_{1} \cos (\alpha \Delta x)+1\right)}{\left(2 R_{1}+d \tau+1\right)}, \\
& \frac{T(t+\Delta t)}{T(t)}=\frac{\left(-4 R_{1} \sin ^{2}\left(\frac{\alpha \Delta x}{2}\right)+1+2 R_{1}\right)}{\left(2 R_{1}+1+d \tau\right)}, \\
& \left|\frac{T(t+\Delta t)}{T(t)}\right|=\left|\frac{-4 R_{1} \sin ^{2}\left(\frac{\alpha \Delta x}{2}\right)+1+2 R_{1}}{1+2 R_{1}+d \tau}\right| \leq\left|\frac{1-2 R_{1}}{1+2 R_{1}+d \tau}\right|<1 .
\end{aligned}
$$

Similarly, following the same lines, (33) gives

$$
\left|\frac{T(t+\Delta t)}{T(t)}\right|=\left|\frac{-4 R_{2} \sin ^{2}\left(\frac{\alpha \Delta x}{2}\right)+1+2 R_{2}}{2 R_{2}+1}\right| \leq\left|\frac{1-2 R_{2}}{1+2 R_{2}}\right|<1 .
$$


From (40) and (41), it is clear that the proposed nonstandard finite difference technique for system (32) is completely stable.

\subsection{Consistency of nonstandard finite difference method}

To check the uniformity of nonstandard finite difference technique, Taylor series is used. The series of $\left.u\right|_{i} ^{j+1},\left.u\right|_{i+1} ^{j}$ and $\left.u\right|_{i-1} ^{j}$ are given below:

$$
\begin{aligned}
& -\left.u\right|_{i} ^{j}+\left.u\right|_{i} ^{j+1}=\left.u_{\tau}\right|_{i} ^{j} d \tau+\left.\frac{(d \tau)^{2}}{2 !} u_{\tau \tau}\right|_{i} ^{j}+\left.\frac{(d \tau)^{3}}{3 !} u_{\tau \tau \tau}\right|_{i} ^{j}+\cdots, \\
& \left.u\right|_{i+1} ^{j}-\left.u\right|_{i} ^{j}=\left.d x u_{x}\right|_{i} ^{j}+\left.\frac{(d x)^{2}}{2 !} u_{x x}\right|_{i} ^{j}+\left.\frac{(d x)^{3}}{3 !} u_{x x x}\right|_{i} ^{j}+\cdots, \\
& \left.u\right|_{i-1} ^{j}-\left.u\right|_{i} ^{j}=-\left.d x u_{x}\right|_{i} ^{j}+\left.\frac{(d x)^{2}}{2 !} u_{x x}\right|_{i} ^{j}-\left.\frac{(d x)^{3}}{3 !} u_{x x x}\right|_{i} ^{j}+\cdots .
\end{aligned}
$$

Consider the unconditional proposed nonstandard finite difference scheme

$$
u_{i}^{j+1}=u_{i}^{j}+\frac{\kappa_{1} d \tau}{(d x)^{2}}\left(u_{i-1}^{j}-2 u_{i}^{j}+u_{i+1}^{j}\right)+l d \tau-u_{i}^{j} d \tau-\frac{4 u_{i}^{j} v_{i}^{j} d \tau}{1+\left(u_{i}^{j}\right)^{2}}
$$

Substituting the values of $\left.u\right|_{i} ^{j+1},\left.u\right|_{i+1} ^{j}$ and $\left.u\right|_{i-1} ^{j}$ into the above equation and after simplification, we have

$$
\begin{aligned}
& \left(\left.u_{\tau}\right|_{i} ^{j}+\left.\frac{d \tau}{2 !} u_{\tau \tau}\right|_{i} ^{j}+\left.\frac{(d \tau)^{2}}{3 !} u_{\tau \tau \tau}\right|_{i} ^{j}+\cdots\right)\left(1+\frac{2 \kappa_{1} d \tau}{(d x)^{2}}+d \tau+\frac{4 v_{i}^{j} d \tau}{1+\left(u_{i}^{j}\right)^{2}}\right) \\
& =2 \kappa_{1}\left(\frac{\left.u_{x x}\right|_{i} ^{j}}{2 !}+\left.\frac{(d x)^{2}}{4 !} u_{x x x x}\right|_{i} ^{j}\right)+l-u_{i}^{j}-\frac{4 u_{i}^{j} v_{i}^{j} d \tau}{1+\left(u_{i}^{j}\right)^{2}} .
\end{aligned}
$$

Putting $d \tau=(d x)^{3}$ and letting $d x \rightarrow 0$, the above equation gives

$$
u_{\tau}=\kappa_{1} \Delta u+l-u-\frac{4 u v}{1+u^{2}} \text {. }
$$

Similarly, Taylor series expansion of $\left.\nu\right|_{i} ^{j+1},\left.v\right|_{i+1} ^{j}$ and $\left.v\right|_{i-1} ^{j}$ are given below:

$$
\begin{aligned}
& -\left.v\right|_{i} ^{j}+\left.v\right|_{i} ^{j+1}=\left.v_{\tau}\right|_{i} ^{j} d \tau+\left.v_{\tau \tau}\right|_{i} ^{j} \frac{(d \tau)^{2}}{2 !}+\left.v_{\tau \tau \tau}\right|_{i} ^{j} \frac{(d \tau)^{3}}{3 !}+\cdots, \\
& -\left.v\right|_{i} ^{j}+\left.v\right|_{i+1} ^{j}=\left.v_{x}\right|_{i} ^{j} d x+\left.v_{x x}\right|_{i} ^{j} \frac{(d x)^{2}}{2 !}+\left.\frac{(d x)^{3}}{3 !} v_{x x x}\right|_{i} ^{j}+\cdots, \\
& -\left.v\right|_{i} ^{j}+\left.v\right|_{i-1} ^{j}=-\left.v_{x}\right|_{i} ^{j} d x+\left.v_{x x}\right|_{i} ^{j} \frac{(d x)^{2}}{2 !}-\left.\frac{(d x)^{3}}{3 !} v_{x x x}\right|_{i} ^{j}+\cdots .
\end{aligned}
$$

Substituting the values of $\left.v\right|_{i} ^{j+1},\left.v\right|_{i+1} ^{j}$ and $\left.v\right|_{i-1} ^{j}$ into equation (33) and after simplification, we get

$$
\begin{aligned}
& \left(\left.v_{\tau}\right|_{i} ^{j}+\left.\frac{d \tau}{2 !} v_{\tau \tau}\right|_{i} ^{j}+\left.\frac{(d \tau)^{2}}{3 !} v_{\tau \tau \tau}\right|_{i} ^{j}+\cdots\right)\left(1+\frac{2 \kappa_{2} d \tau}{(d x)^{2}}+m \frac{u_{i}^{j} d \tau}{1+\left(u_{i}^{j}\right)^{2}}\right) \\
& =2 \kappa_{2}\left(\frac{\left.v_{x x}\right|_{i} ^{j}}{2 !}+\left.\frac{(d x)^{2}}{4 !} v_{x x x x}\right|_{i} ^{j}\right)+m u_{i}^{j}-\frac{m u_{i}^{j} v_{i}^{j}}{1+\left(u_{i}^{j}\right)^{2}} .
\end{aligned}
$$


Putting $d \tau=(d x)^{3}$ and letting $d x \rightarrow 0$, the above equation gives

$$
v_{\tau}=\kappa_{2} \Delta v+m\left(u-\frac{u}{1+u^{2}} v\right)
$$

\section{Second model}

In this second example, we assume $\psi(u)=u\left(1+e^{u}\right)^{-1}$, then the system (7) takes the form

$$
\left\{\begin{array}{l}
u_{\tau}=\kappa_{1} \Delta u+l-u-\frac{4 u v}{1+e^{u}}, \\
v_{\tau}=\kappa_{2} \Delta v+m\left(u-\frac{u v}{1+e^{u}}\right) .
\end{array}\right.
$$

The equilibrium point of this system is $\left(\mu, 1+e^{\mu}\right)$. To discretize the system (45) using finite difference method, firstly $[0, \Im]^{2} \times[0, \mathrm{~T}]$ is divided into $M^{2} \times N$ parts with spatial and temporal step sizes $d x=\frac{\Im}{M}$ and $d \tau=\frac{T}{N}$. Then the grid points are $x_{i}=i d x$ and $\tau_{j}=j d \tau$, where $i=0,1,2, \ldots, M$ and $j=0,1,2, \ldots, N$.

\subsection{Forward Euler method}

Substituting the values of $\left.u_{\tau}\right|_{i} ^{j}$ and $\left.u_{x x}\right|_{i} ^{j}$ into the first equation of (45) and the values of $\left.v_{\tau}\right|_{i} ^{j}$ and $\left.v_{x x}\right|_{i} ^{j}$ into the second equation of (45), we have

$$
\begin{aligned}
& \delta_{\tau} u_{i}^{j}=\frac{\kappa_{1}}{2} \delta_{x}^{2}\left(u_{i}^{j}+u_{i}^{j+1}\right)+l-u_{i}^{j}-\frac{4 u_{i}^{j} v_{i}^{j}}{1+e^{u_{i}^{j}}}, \\
& \delta_{\tau} v_{i}^{j}=\frac{\kappa_{2}}{2} \delta_{x}^{2}\left(v_{i}^{j}+v_{i}^{j+1}\right)+m\left(u_{i}^{j}-\frac{u_{i}^{j} v_{i}^{j}}{1+e^{u_{i}^{j}}}\right) .
\end{aligned}
$$

After simple calculation, we obtain

$$
\begin{aligned}
& u_{i}^{j+1}=R_{1}\left(u_{i-1}^{j}-2 u_{i}^{j}+u_{i+1}^{j}\right)+u_{i}^{j}+l d \tau-u_{i}^{j} d \tau-\frac{4 u_{i}^{j} v_{i}^{j} d \tau}{1+e^{u_{i}^{j}}}, \\
& v_{i}^{j+1}=R_{2}\left(v_{i-1}^{j}-2 v_{i}^{j}+v_{i+1}^{j}\right)+v_{i}^{j}+m d \tau-\frac{m u_{i}^{j} v_{i}^{j} d \tau}{1+e^{u_{i}^{j}}},
\end{aligned}
$$

where $R_{1}=\frac{\kappa_{1} d \tau}{(d x)^{2}}$ and $R_{2}=\frac{\kappa_{2} d \tau}{(d x)^{2}}$. This technique is conditionally stable for $\frac{2-d \tau}{4}>R_{1}$ and $\frac{1}{2}>R_{2}$ for the structure (45).

\subsection{Crank-Nicolson method}

Substituting the values of $\left.u_{\tau}\right|_{i} ^{j},\left.u_{x x}\right|_{i} ^{j}$ and $\left.u_{x x}\right|_{i} ^{j+1}$ into the first equation of (45) and the values of $\left.v_{\tau}\right|_{i} ^{j},\left.v_{x x}\right|_{i} ^{j}$ and $\left.v_{x x}\right|_{i} ^{j+1}$ into the second equation of (30), we have

$$
\begin{aligned}
& \delta_{\tau} u_{i}^{j}=\frac{\kappa_{1}}{2} \delta_{x}^{2}\left(u_{i}^{j}+u_{i}^{j+1}\right)+l-u_{i}^{j}-\frac{4 u_{i}^{j} v_{i}^{j}}{1+e^{u_{i}^{j}}}, \\
& \delta_{\tau} v_{i}^{j}=\frac{\kappa_{2}}{2} \delta_{x}^{2}\left(v_{i}^{j}+v_{i}^{j+1}\right)+m\left(u_{i}^{j}-\frac{u_{i}^{j} v_{i}^{j}}{1+e^{u_{i}^{j}}}\right) .
\end{aligned}
$$


After simple calculation, we have

$$
\begin{aligned}
u_{i}^{j+1} & -\frac{R_{1}}{2}\left(u_{i+1}^{j+1}-2 u_{i}^{j+1}+u_{i-1}^{j+1}\right) \\
& =\frac{R_{1}}{2}\left(u_{i-1}^{j}-2 u_{i}^{j}+u_{i+1}^{j}\right)+u_{i}^{j}+l d \tau-u_{i}^{j} d \tau-\frac{4 u_{i}^{j} v_{i}^{j} d \tau}{1+e^{u_{i}^{j}}}, \\
v_{i}^{j+1} & -\frac{R_{2}}{2}\left(v_{i+1}^{j+1}-2 v_{i}^{j+1}+v_{i-1}^{j+1}\right) \\
& =\frac{R_{2}}{2}\left(v_{i-1}^{j}-2 v_{i}^{j}+v_{i+1}^{j}\right)+v_{i}^{j}+m u_{i}^{j} d \tau-\frac{m u_{i}^{j} v_{i}^{j} d \tau}{1+e^{u_{i}^{j}}},
\end{aligned}
$$

where $R_{1}=\frac{\kappa_{1} d \tau}{(d x)^{2}}$ and $R_{2}=\frac{\kappa_{2} d \tau}{(d x)^{2}}$. This technique is completely stable for the structure (45).

\subsection{Proposed nonstandard finite difference method}

Here, we will present a scheme the nonstandard finite difference method for the system (45). Substituting the values of $\left.u_{\tau}\right|_{i} ^{j},\left.u_{x x}\right|_{i} ^{j},\left.v_{\tau}\right|_{i} ^{j}$ and $\left.v_{x x}\right|_{i} ^{j}$ into equation (53), we have

$$
\begin{aligned}
& \frac{u_{i}^{j+1}-u_{i}^{j}}{d \tau}=l-u_{i}^{j}-\frac{4 u_{i}^{j} v_{i}^{j}}{1+e^{u_{i}^{j}}}+\frac{\kappa_{1}}{(d x)^{2}}\left(u_{i-1}^{j}-2 u_{i}^{j}+u_{i+1}^{j}\right), \\
& \frac{v_{i}^{j+1}-v_{i}^{j}}{d \tau}=m u_{i}^{j}-\frac{m u_{i}^{j} v_{i}^{j}}{1+e^{u_{i}^{j}}}+\frac{\kappa_{2}}{(d x)^{2}}\left(v_{i-1}^{j}-2 v_{i}^{j}+v_{i+1}^{j}\right) .
\end{aligned}
$$

After simple calculation, we have

$$
\left\{\begin{array}{l}
u_{i}^{j+1}=\frac{R_{1}\left(u_{i-1}^{j}+u_{i+1}^{j}\right)+u_{i}^{j}+l d \tau}{\left(1+2 R_{1}+d \tau+\frac{4 v_{i}^{j} d \tau}{j u^{j}}\right)}, \\
v_{i}^{j+1}=\frac{v_{i}^{j}+R_{2}\left(v_{i-1}^{j}+v_{i+1}^{j}\right)+m u_{i}^{j} d \tau}{\left(1+2 R_{2}+\frac{m u_{i}^{j} d \tau}{u_{i}^{j}}\right)}, \\
1+e^{u_{i}}
\end{array}\right.
$$

where $R_{1}=\frac{\kappa_{1} d \tau}{(d x)^{2}}$ and $R_{2}=\frac{\kappa_{2} d \tau}{(d x)^{2}}$. This technique is completely stable for the structure (53).

\subsection{Stability analysis of nonstandard finite difference method}

To find the stability bounds, the von Neumann stability technique is used. After linearizing equation (31) and then using the von Neumann stability bounds, we get

$$
\begin{aligned}
& T(t+\Delta t) e^{i \alpha x}-T(t) e^{i \alpha x}=R_{1}\left(-2 T(t+\Delta t) e^{i \alpha x}+T(t) e^{i \alpha(x-\Delta x)}+T(t) e^{i \alpha(x+\Delta x)}\right) \\
& -d \tau e^{i \alpha x} T(t+\Delta t) \\
& \left(1+2 R_{1}+d \tau\right) e^{i \alpha x} T(t+\Delta t)=\left(1+2 R_{1} \cos (\alpha \Delta x)\right) e^{i \alpha x} T(t), \\
& \frac{T(\Delta t+t)}{T(t)}=\frac{\left(2 R_{1} \cos (\alpha \Delta x)+1\right)}{\left(1+2 R_{1}+d \tau\right)}, \\
& \frac{T(t+\Delta t)}{T(t)}=\frac{\left(1+2 R_{1}-4 R_{1} \sin ^{2}\left(\frac{\alpha \Delta x}{2}\right)\right)}{\left(2 R_{1}+1+d \tau\right)},
\end{aligned}
$$




$$
\left|\frac{T(t+\Delta t)}{T(t)}\right|=\left|\frac{-4 R_{1} \sin ^{2}\left(\frac{\alpha \Delta x}{2}\right)+1+2 R_{1}}{2 R_{1}+d \tau+1}\right| \leq\left|\frac{1-2 R_{1}}{1+2 R_{1}+d \tau}\right|<1
$$

Similarly, following the same lines, (52) gives

$$
\left|\frac{T(t+\Delta t)}{T(t)}\right|=\left|\frac{-4 R_{2} \sin ^{2}\left(\frac{\alpha \Delta x}{2}\right)+1+2 R_{2}}{2 R_{2}+1}\right| \leq\left|\frac{1-2 R_{2}}{1+2 R_{2}}\right|<1 .
$$

From (54) and (55), it is clear that the proposed nonstandard finite difference technique for system (32) is unconditionally stable.

\subsection{Consistency of nonstandard finite difference method}

To check the uniformity of the nonstandard finite difference technique, Taylor series is used. The series of $\left.u\right|_{i} ^{j+1},\left.u\right|_{i+1} ^{j}$ and $\left.u\right|_{i-1} ^{j}$ are given below:

$$
\begin{aligned}
& -\left.u\right|_{i} ^{j}+\left.u\right|_{i} ^{j+1}=\left.u_{\tau}\right|_{i} ^{j} d \tau+\left.u_{\tau \tau}\right|_{i} ^{j} \frac{(d \tau)^{2}}{2 !}+\left.u_{\tau \tau \tau}\right|_{i} ^{j} \frac{(d \tau)^{3}}{3 !}+\cdots, \\
& -\left.u\right|_{i} ^{j}+\left.u\right|_{i+1} ^{j}=\left.u_{x}\right|_{i} ^{j} d x+\left.u_{x x}\right|_{i} ^{j} \frac{(d x)^{2}}{2 !}+\left.u_{x x x}\right|_{i} ^{j} \frac{(d x)^{3}}{3 !}+\cdots, \\
& -\left.u\right|_{i} ^{j}+\left.u\right|_{i-1} ^{j}=-\left.u_{x}\right|_{i} ^{j} d x+\left.u_{x x}\right|_{i} ^{j} \frac{(d x)^{2}}{2 !}-\left.u_{x x x}\right|_{i} ^{j} \frac{(d x)^{3}}{3 !}+\cdots .
\end{aligned}
$$

Consider the unconditional proposed nonstandard finite difference scheme

$$
\begin{aligned}
& u_{i}^{j+1}=\frac{\kappa_{1} d \tau}{(d x)^{2}}\left(u_{i-1}^{j}-2 u_{i}^{j}+u_{i+1}^{j}\right)+u_{i}^{j}+l d \tau-u_{i}^{j} d \tau-\frac{4 u_{i}^{j} v_{i}^{j} d \tau}{1+e^{u_{i}^{j}}}, \\
& \left(\left.u_{\tau}\right|_{i} ^{j}+\left.u_{\tau \tau}\right|_{i} ^{j} \frac{d \tau}{2 !}+\left.u_{\tau \tau \tau}\right|_{i} ^{j} \frac{(d \tau)^{2}}{3 !}+\cdots\right)\left(1+\frac{2 \kappa_{1} d \tau}{(d x)^{2}}+d \tau+\frac{4 v_{i}^{j} d \tau}{1+\left(u_{i}^{j}\right)^{2}}\right) \\
& =2 \kappa_{1}\left(\frac{\left.u_{x x}\right|_{i} ^{j}}{2 !}+\left.\frac{(d x)^{2}}{4 !} u_{x x x x}\right|_{i} ^{j}\right)+l-u_{i}^{j}-\frac{4 u_{i}^{j} v_{i}^{j} d \tau}{1+e^{u_{i}^{j}}} .
\end{aligned}
$$

Putting $d \tau=(d x)^{3}$ and letting $d x \rightarrow 0$, the above equation gives

$$
u_{\tau}=\kappa_{1} \Delta u+l-u-\frac{4 u v}{1+e^{u}} .
$$

Similarly, Taylor series expansion of $\left.v\right|_{i} ^{j+1},\left.v\right|_{i+1} ^{j}$ and $\left.v\right|_{i-1} ^{j}$ are given below:

$$
\begin{aligned}
& -\left.v\right|_{i} ^{j}+\left.v\right|_{i} ^{j+1}=\left.v_{\tau}\right|_{i} ^{j} d \tau+\left.v_{\tau \tau}\right|_{i} ^{j} \frac{(d \tau)^{2}}{2 !}+\left.v_{\tau \tau \tau}\right|_{i} ^{j} \frac{(d \tau)^{3}}{3 !}+\cdots, \\
& -\left.v\right|_{i} ^{j}+\left.v\right|_{i+1} ^{j}=\left.v_{x}\right|_{i} ^{j} d x+\left.v_{x x}\right|_{i} ^{j} \frac{(d x)^{2}}{2 !}+\left.v_{x x x}\right|_{i} ^{j} \frac{(d x)^{3}}{3 !}+\cdots, \\
& -\left.v\right|_{i} ^{j}+\left.v\right|_{i-1} ^{j}=-\left.v_{x}\right|_{i} ^{j} d x+\left.v_{x x}\right|_{i} ^{j} \frac{(d x)^{2}}{2 !}-\left.v_{x x x}\right|_{i} ^{j} \frac{(d x)^{3}}{3 !}+\cdots .
\end{aligned}
$$


Substituting the values of $\left.v\right|_{i} ^{j+1},\left.v\right|_{i+1} ^{j}$ and $\left.v\right|_{i-1} ^{j}$ into equation (32) and after simplification, we get

$$
\begin{aligned}
& \left(\left.v_{\tau}\right|_{i} ^{j}+\left.v_{\tau \tau}\right|_{i} ^{j} \frac{d \tau}{2 !}+\left.v_{\tau \tau \tau}\right|_{i} ^{j} \frac{(d \tau)^{2}}{3 !}+\cdots\right)\left(1+\frac{2 \kappa_{2} d \tau}{(d x)^{2}}+m \frac{u_{i}^{j} d \tau}{1+e^{u_{i}^{j}}}\right) \\
& =2 \kappa_{2}\left(\frac{\left.v_{x x}\right|_{i} ^{j}}{2 !}+\left.\frac{(d x)^{2}}{4 !} v_{x x x x}\right|_{i} ^{j}\right)+m u_{i}^{j}-\frac{m u_{i}^{j} v_{i}^{j}}{1+e^{u_{i}^{j}}} .
\end{aligned}
$$

Putting $d \tau=(d x)^{3}$ and letting $d x \rightarrow 0$, the above equation gives $v_{\tau}=\kappa_{2} \Delta v+m\left(u-\frac{u v}{1+e^{u}}\right)$.

\section{Results and discussion}

\subsection{Test Problem 1}

Here, the proposed (i) forward Euler scheme, (ii) Crank-Nicolson scheme, and (iii) nonstandard finite difference scheme are tested on the model considered for the onedimensional CIMA problem

$$
\left\{\begin{array}{l}
u_{\tau}=l-u-4 \frac{u}{1+u^{2}} v+\kappa_{1} \Delta u, \\
v_{\tau}=m\left(u-\frac{u}{1+u^{2}} v\right)+\kappa_{2} \Delta v,
\end{array} \quad \text { with } u_{0}=1+\cos (x), v_{0}=2+\sin (x) .\right.
$$

Numerical simulations are carried out to confirm the efficiency and effectiveness of the nonstandard finite difference method. Figures $1(\mathrm{a})-(\mathrm{d}), 3(\mathrm{a})-(\mathrm{d})$, and 5(a)-(d) represent the 2D concentration and mesh plots for $u(x, \tau)$ and $v(x, \tau)$ using forward Euler, CrankNicolson and nonstandard finite difference schemes with $l=5, m=1, \kappa_{1}=0.01$, and $\kappa_{2}=0.01$. In all figures, the system converges to the constant steady state (Figs. 1(a)-(d), $1(\mathrm{e})-(\mathrm{h}))$. Figures $1(\mathrm{e})-(\mathrm{h}), 2(\mathrm{e})-(\mathrm{h})$, and 3(e)-(h) represent the mesh plots and projected views for $u(x, \tau)$ and $v(x, \tau)$. Mesh plots and projected views of Figs. $1(\mathrm{e})-(\mathrm{f}), 2(\mathrm{e})-(\mathrm{h})$, and $3(\mathrm{e})-(\mathrm{h})$ indicate that the solutions $u(x, \tau)$ and $v(x, \tau)$ tend to a spatially homogeneous periodic orbit. The stripes of Fig. 3(e)-(h) fade away whereas the stripes of Figs. 1(a) -(d) and 2(e)-(h) remain for the same time $\tau=20$. It has been observed that when we increase the value of $d \tau$ by 0.5 , the forward Euler and Crank-Nicolson schemes fail to provide stable solutions (Figs. 7(a)-(d) and 7(e)-(h)), whereas the explicit nonstandard finite difference scheme provides stable solutions even for $d \tau=0.1,0.5,1,10,100$.

\subsection{Test Problem 2}

Here, the proposed (i) forward Euler scheme, (ii) Crank-Nicolson scheme, and (iii) nonstandard finite difference scheme are tested on a model described by the one-dimensional problem as

$$
\left\{\begin{array}{l}
u_{\tau}=l-u-\frac{4 u v}{1+e^{u}}+\kappa_{1} \Delta u, \\
v_{\tau}=m\left(u-\frac{u v}{1+e^{u}}\right)+\kappa_{2} \Delta v,
\end{array} \quad \text { with } u_{0}=1+\cos (x) \text { and } v_{0}=2+\sin (x)\right.
$$

Numerical simulations are carried out to confirm the efficiency and effectiveness of the nonstandard finite difference method. Figures 4(a)-(d), 5(a)-(d), and 6(a)-(d) represent the $2 \mathrm{D}$ concentration and mesh plots for $u(x, \tau)$ and $v(x, \tau)$ using forward Euler, CrankNicolson, and nonstandard finite difference schemes with $l=5, m=1, \kappa_{1}=0.01$, and 


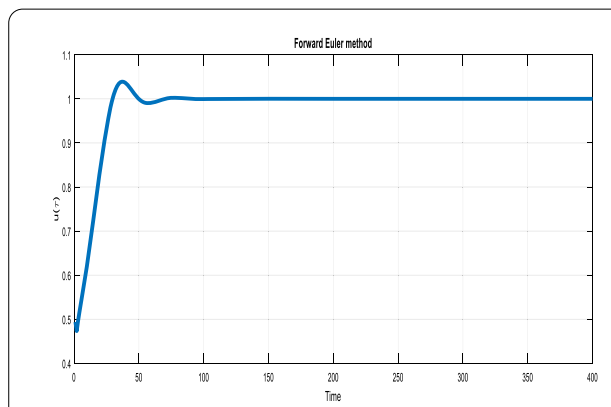

(a)

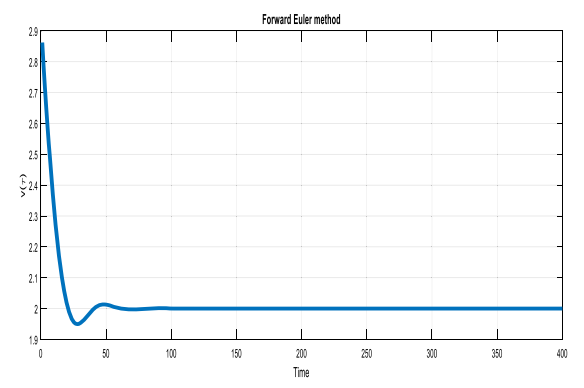

(c)

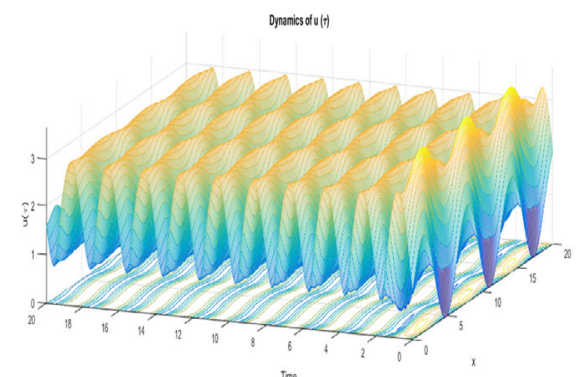

(e)

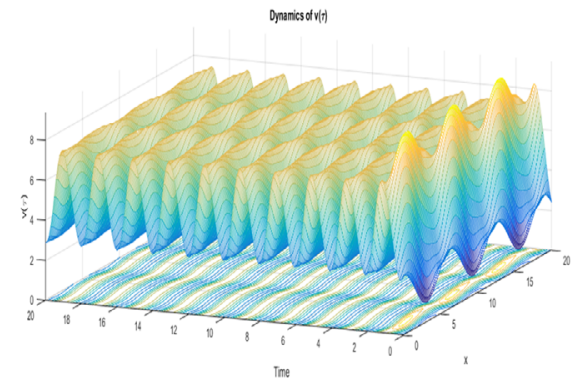

$(g)$

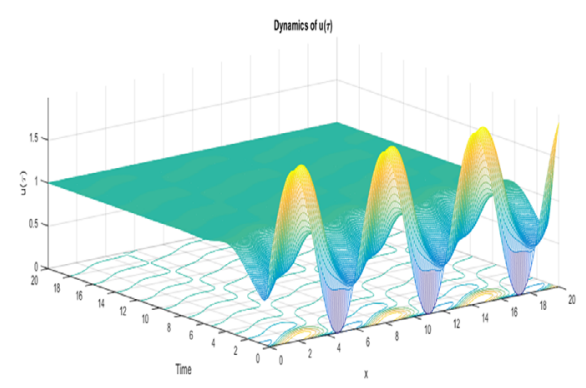

(b)

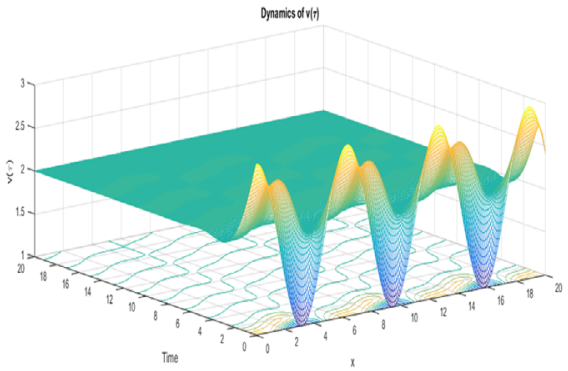

(d)

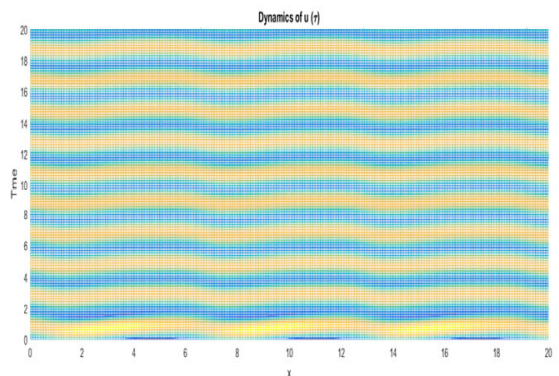

$(f)$

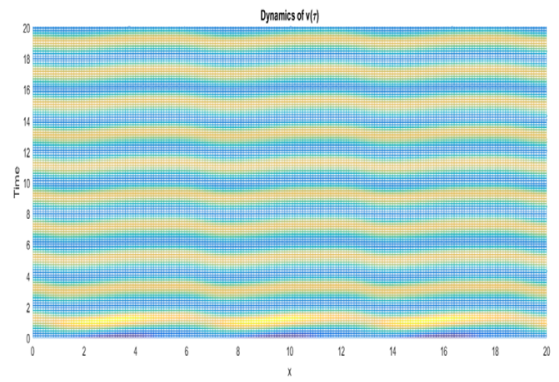

(h)

Figure 1 (a)-(d) Numerical solutions of system (32) using forward Euler scheme. Here, $u_{0}=1+\sin (x)$ and $v_{0}=2+\cos (x)$, with $I=5, m=1, \kappa_{1}=0.01$, and $\kappa_{2}=0.01$. The concentration of $u(\tau)$ is at the top (2D and 3D plots) while the concentration of $v(\tau)$ is at the bottom (2D and $3 D$ plots). The solutions $v(x, \tau)$ and $u(x, \tau)$ tend to the constant steady state. (e)-(h) Numerical solutions of system (32) using forward Euler scheme. Here, $u_{0}=1+\sin (x)$ and $v_{0}=2+\cos (x)$, with $I=10, m=5, \kappa_{1}=0.01$, and $\kappa_{2}=0.01$. (Top) The solutions $v(x, \tau)$ and $u(x, \tau)$ tend to the spatially homogeneous periodic orbit. (Bottom) The projected views onto the $x \tau$-plane at $\tau=20$ for $v(x, \tau)$ and $u(x, \tau)$. The stripe structure invades the homogeneous periodic orbit for $v(x, \tau)$ and $u(x, \tau)$ 


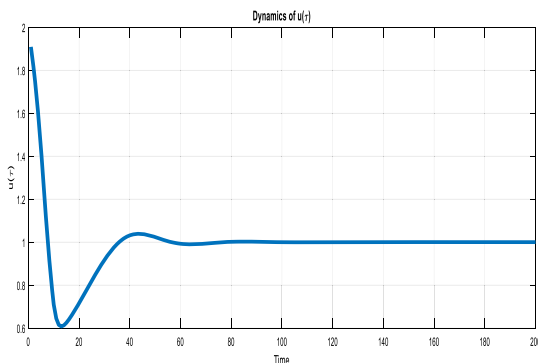

(a)

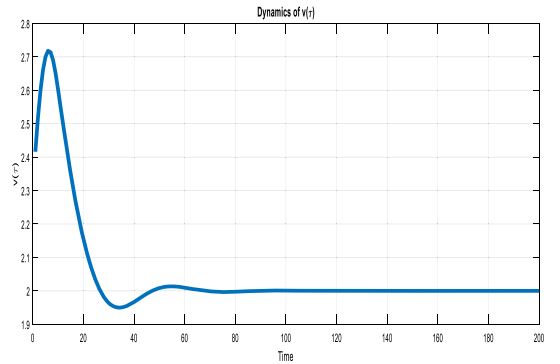

(c)

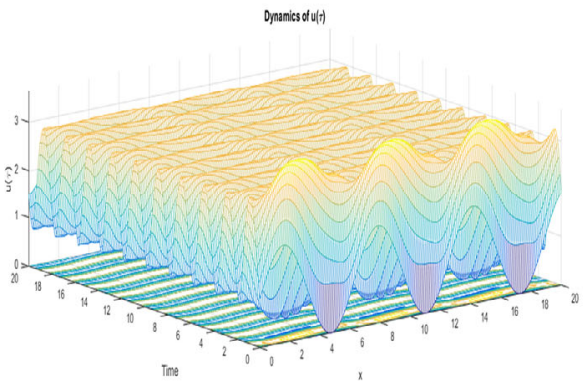

(e)

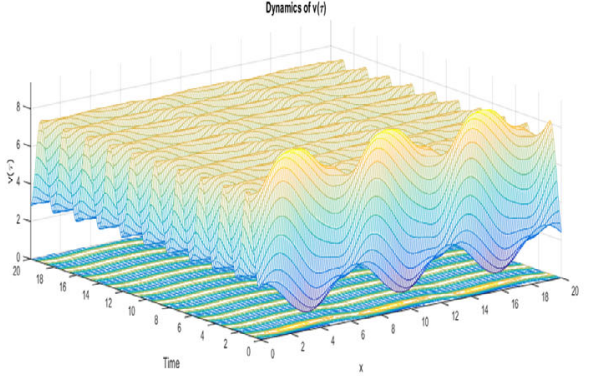

$(g)$

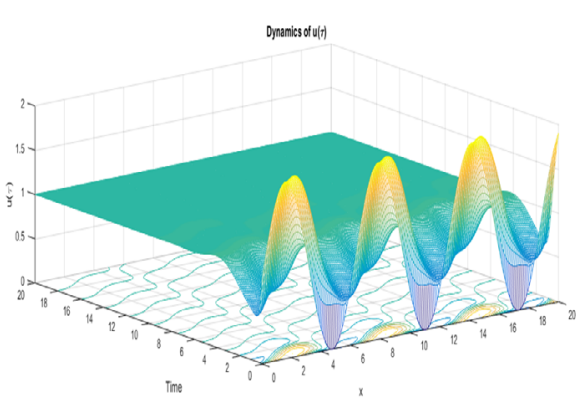

(b)

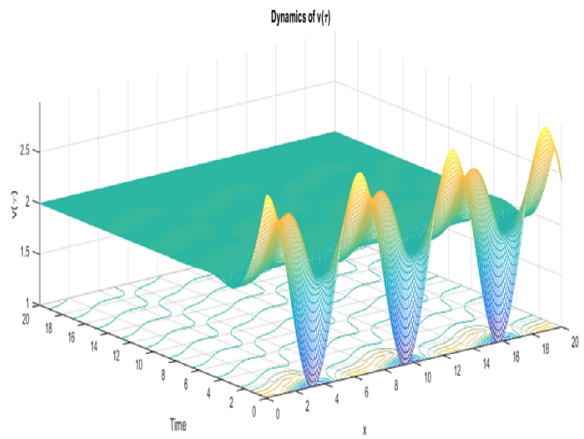

(d)

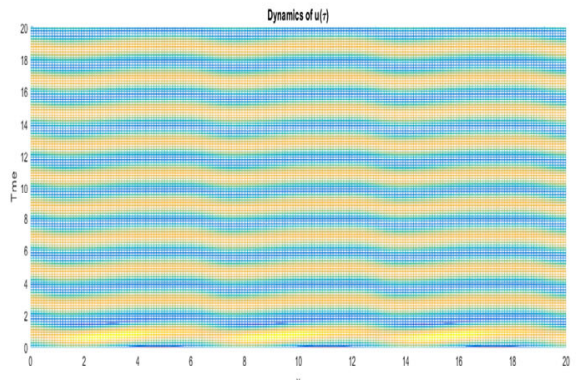

(f)

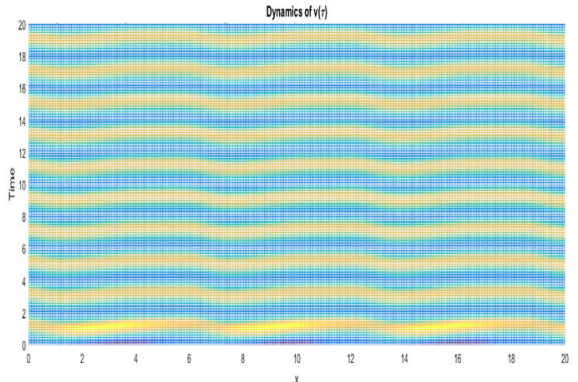

(h)

Figure 2 (a)-(d) Numerical solutions of system (32) using Crank-Nicolson scheme. Here, $u_{0}=1+\sin (x)$ and $v_{0}=2+\cos (x)$, with $l=5, m=1, \kappa_{1}=0.01$, and $\kappa_{2}=0.01$. (Top) The concentration of $u(\tau)$ is on the left while the concentration of $v(\tau)$ is on the right. (Bottom) The solutions $v(x, \tau)$ and $u(x, \tau)$ tend to the constant steady state. (e)-(h) Numerical solutions of system (32) using Crank-Nicolson scheme. Here, $u_{0}=1+\sin (x)$ and $v_{0}=2+\cos (x)$, with $I=10, m=5, \kappa_{1}=0.01$, and $\kappa_{2}=0.01$. (Top) The solutions $v(x, \tau)$ and $u(x, \tau)$ tend to the spatially homogeneous periodic orbit. (Bottom) The projected views onto the $x \tau$-plane at $\tau=20$ for $v(x, \tau)$ and $u(x, \tau)$. The stripe structure invades the homogeneous periodic orbit for $u(x, \tau)$ and $v(x, \tau)$ 


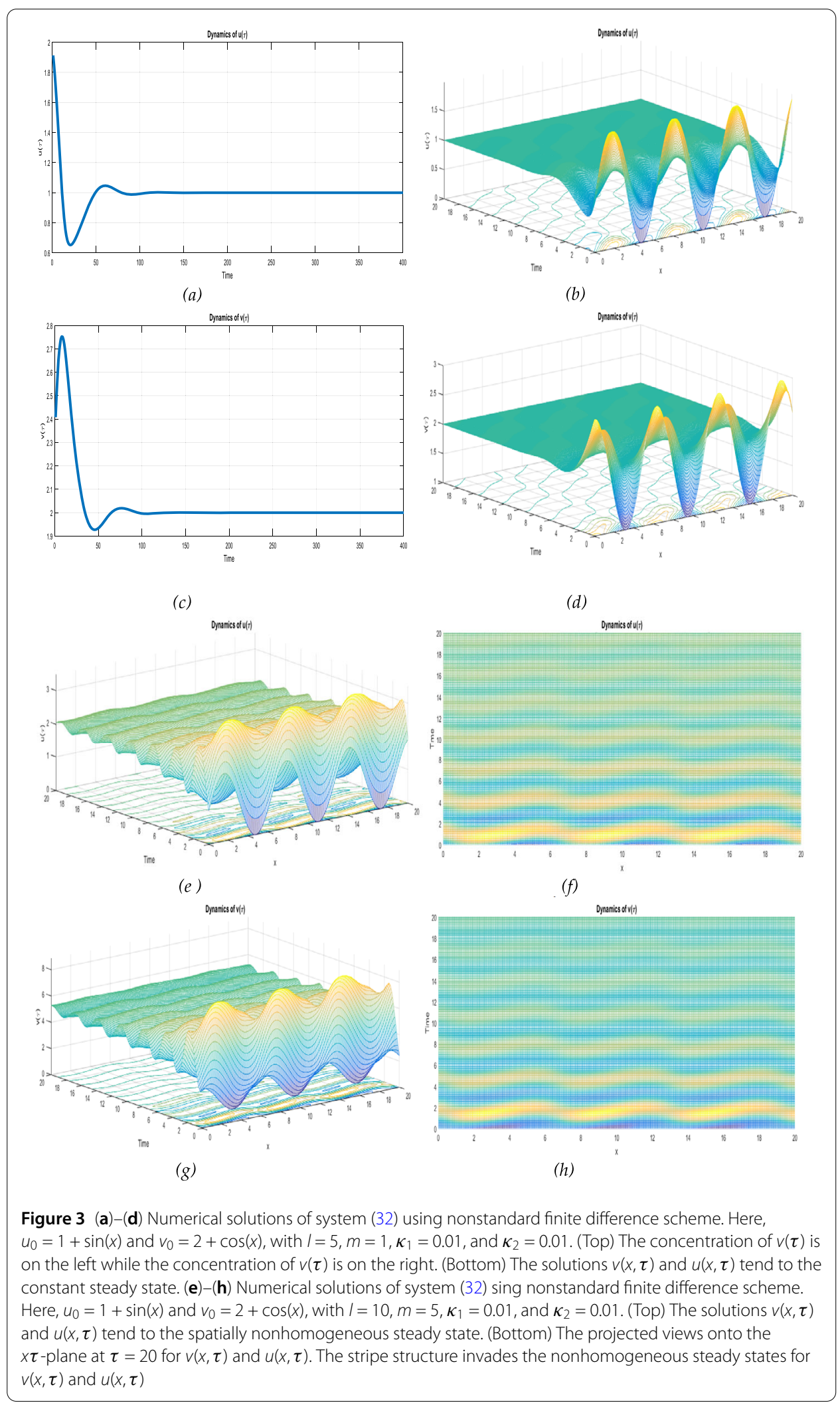




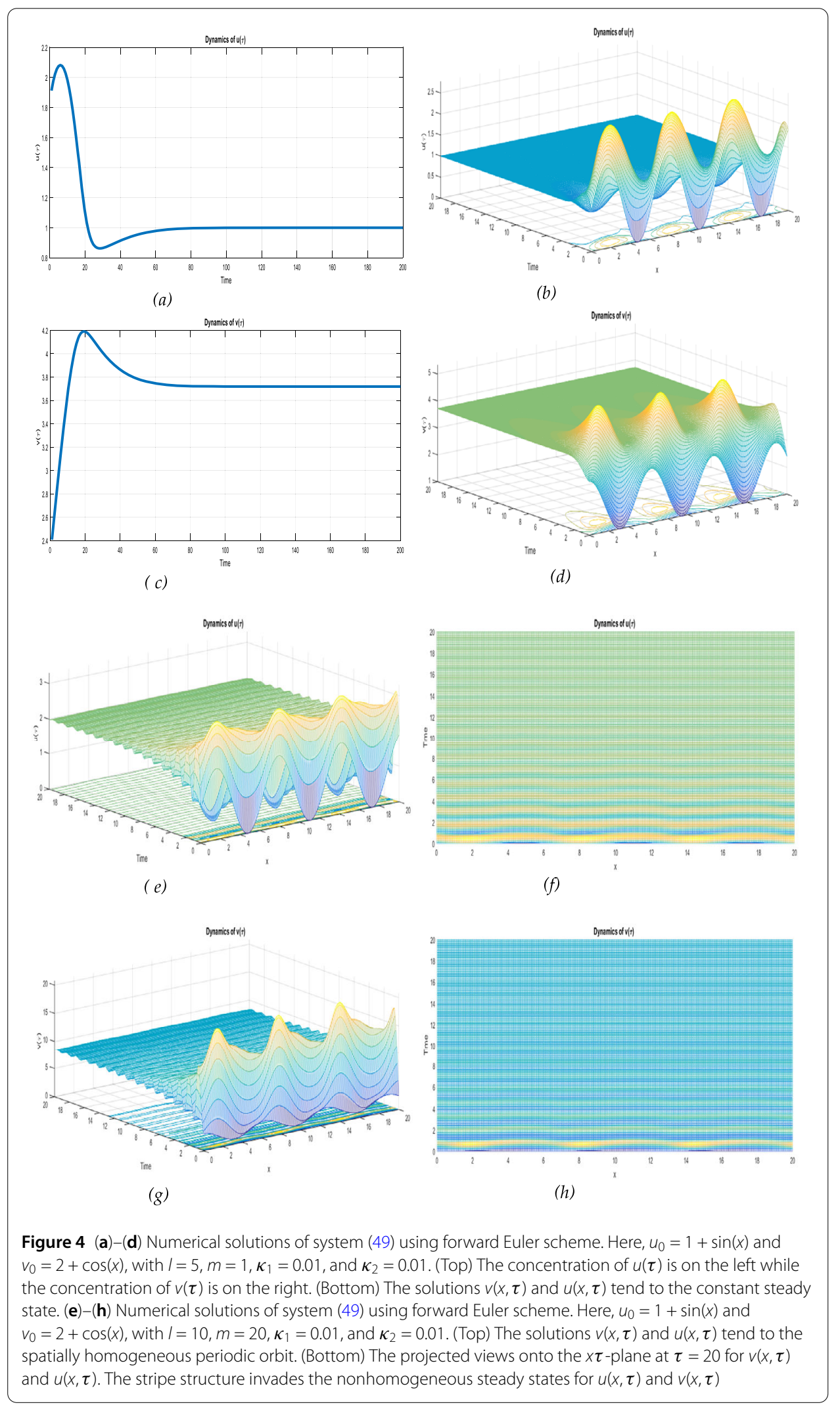




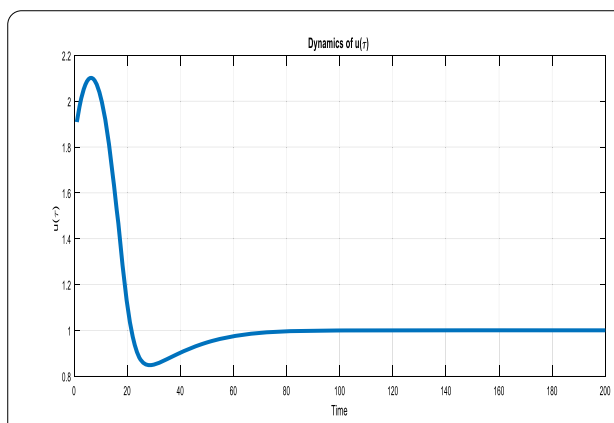

(a)

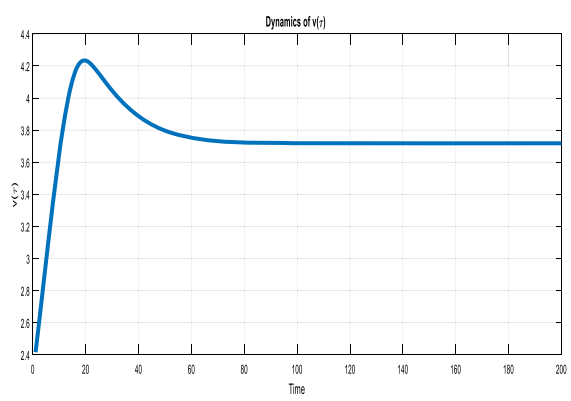

(c)

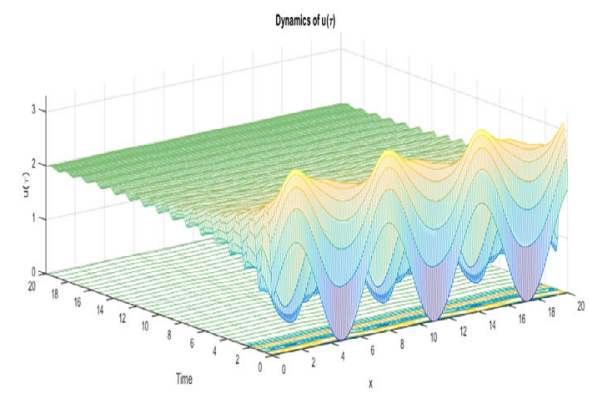

(e)

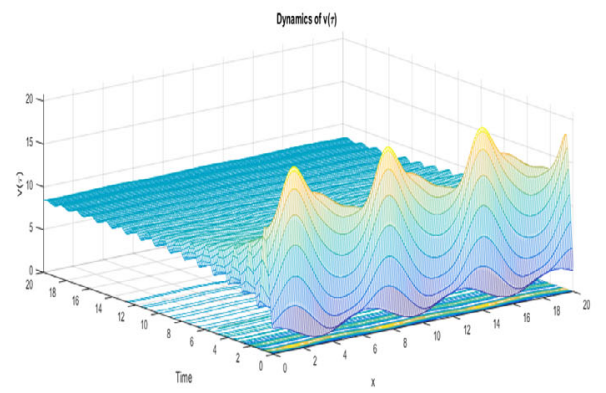

$(g)$

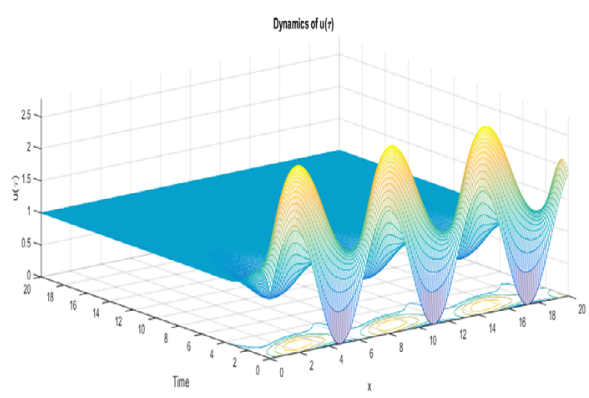

(b)

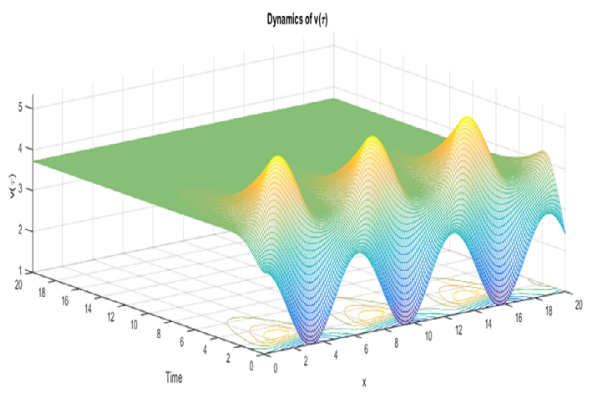

(d)

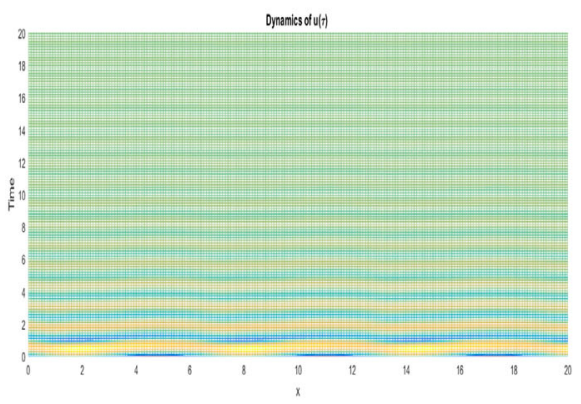

(f)

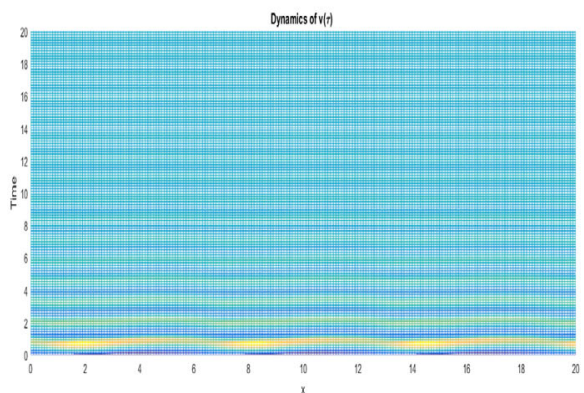

(h)

Figure 5 (a)-(d) Numerical solutions of system (49) using Crank-Nicolson scheme. Here, $u_{0}=1+\sin (x)$ and $v_{0}=2+\cos (x)$, with $I=5, m=1, \kappa_{1}=0.01$, and $\kappa_{2}=0.01$. (Top) The concentration of $u(\tau)$ is on the left while the concentration of $v(\tau)$ is on the right. (Bottom) The solutions $v(x, \tau)$ and $u(x, \tau)$ tend to the constant steady state. (e)-(h) Numerical solutions of system (49) using Crank-Nicolson scheme. Here, $u_{0}=1+\sin (x)$ and $v_{0}=2+\cos (x)$, with $I=10, m=20, \kappa_{1}=0.01$, and $\kappa_{2}=0.01$. (Top) The solutions $v(x, \tau)$ and $u(x, \tau)$ tend to the spatially nonhomogeneous steady states. (Bottom) The projected views onto the $x \tau$-plane at $\tau=20$ for $v(x, \tau)$ and $u(x, \tau)$. The stripe structure invades the nonhomogeneous steady states for $v(x, \tau)$ and $u(x, \tau)$ 


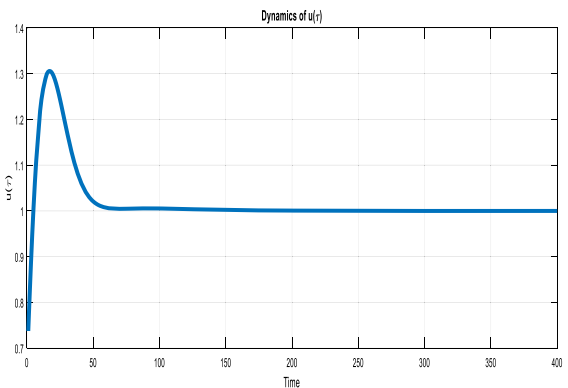

(a)

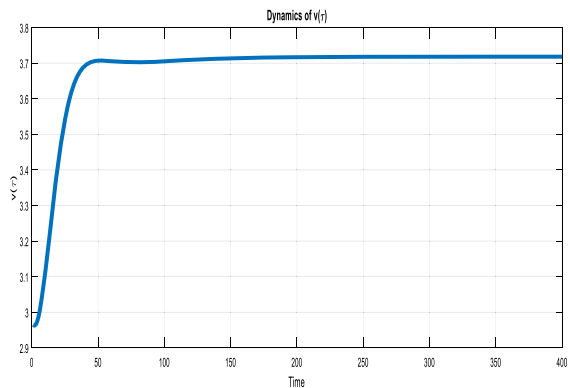

(c)

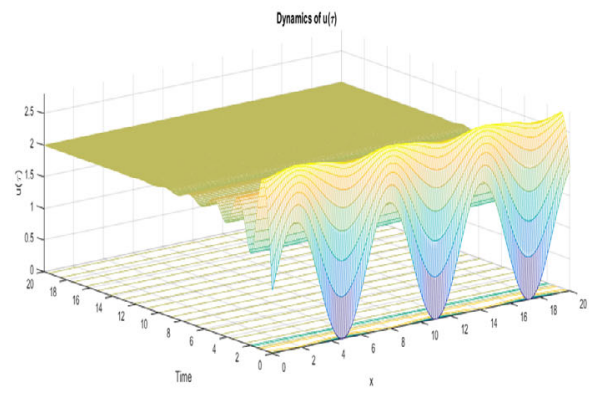

(e)

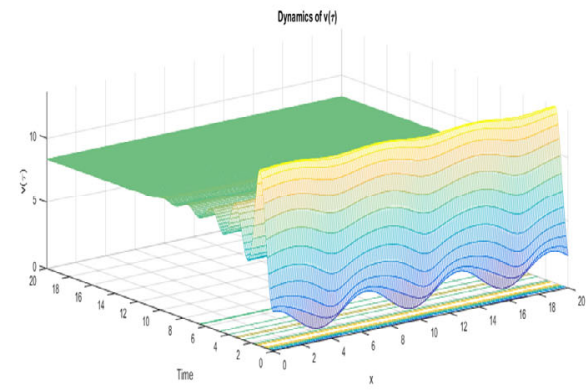

(g)

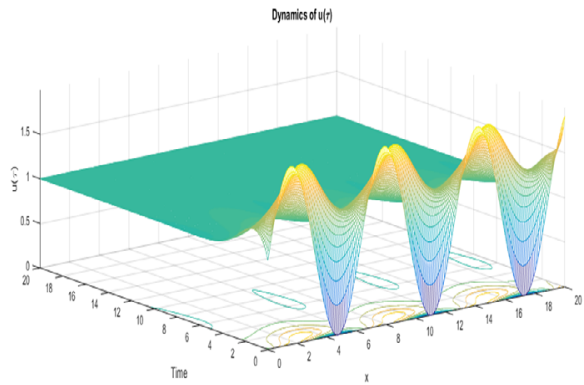

(b)

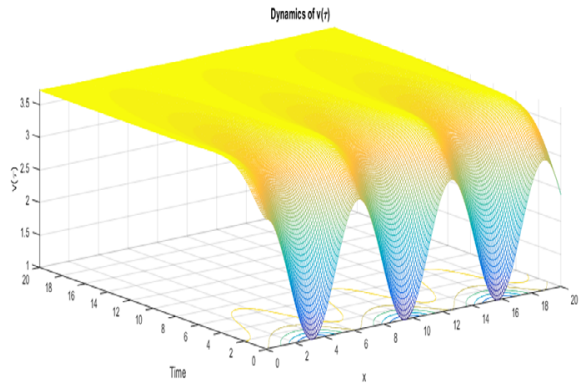

(d)

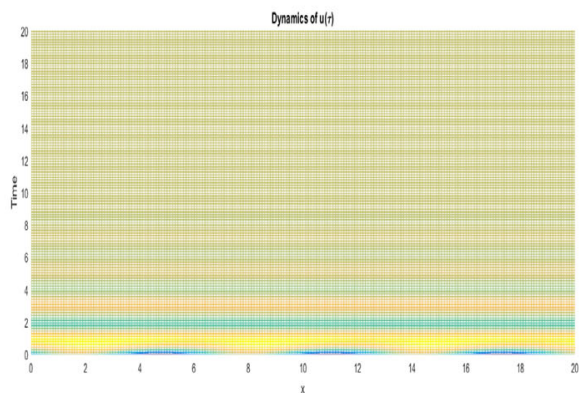

(f)

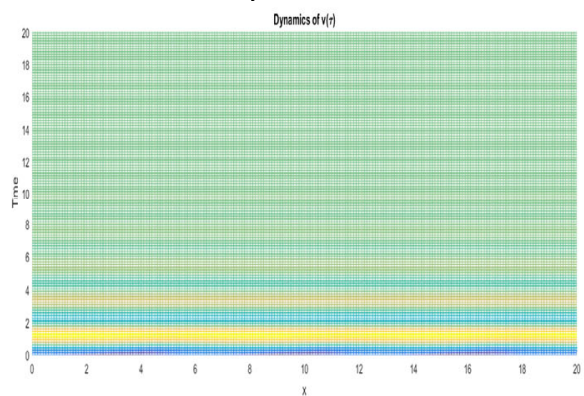

(h)

Figure 6 (a)-(d) Numerical solutions of system (49) using nonstandard finite difference scheme. Here, $u_{0}=1+\sin (x)$ and $v_{0}=2+\cos (x)$, with $/=5, m=1, \kappa_{1}=0.01$, and $\kappa_{2}=0.01$. (Top) The concentration of $u(\tau)$ is on the left while the concentration of $v(\tau)$ is on the right. (Bottom) The solutions $u(x, \tau)$ and $v(x, \tau)$ tend to the constant steady state. (e)-(h) Numerical solutions of system (49) using nonstandard finite difference scheme. Here, $u_{0}=1+\sin (x)$ and $v_{0}=2+\cos (x)$, with $/=10, m=20, \kappa_{1}=0.01$, and $\kappa_{2}=0.01$. (Top) The solutions $v(x, \tau)$ and $u(x, \tau)$ tend to the spatially nonhomogeneous steady state. (Bottom) The projected views onto the $x \tau$-plane at $\tau=20$ for $v(x, \tau)$ and $u(x, \tau)$. The stripe structure invades the nonhomogeneous steady states for $v(x, \tau)$ and $u(x, \tau)$ 


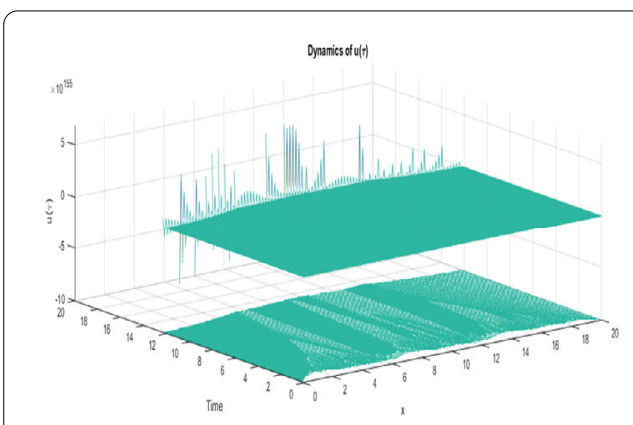

(a)

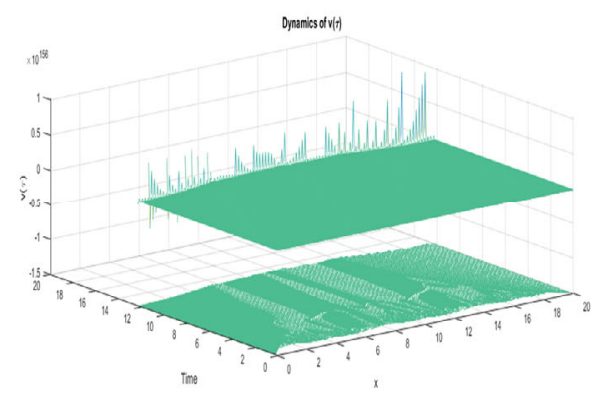

(c)

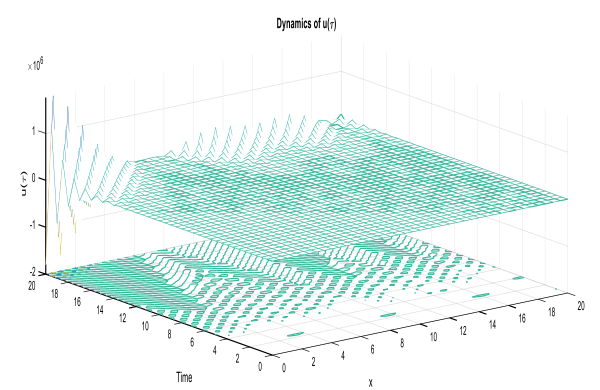

(e)

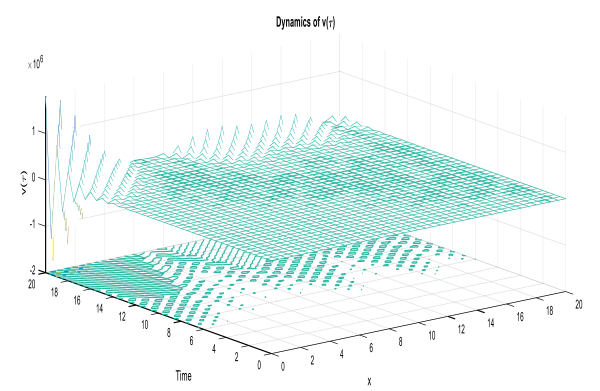

(g)

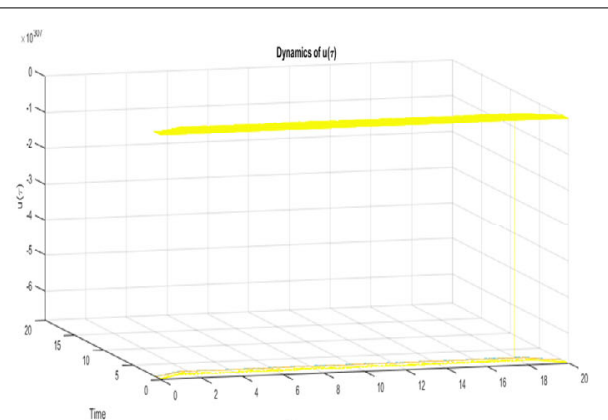

(b)

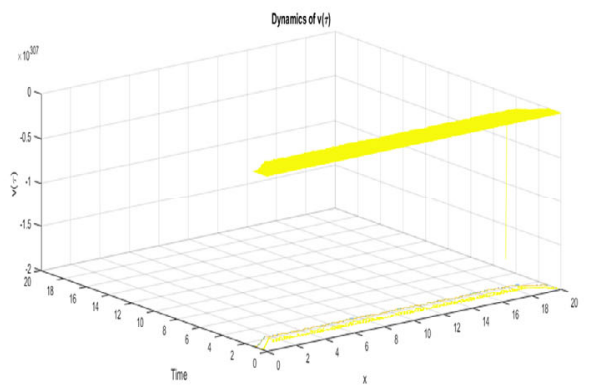

(d)

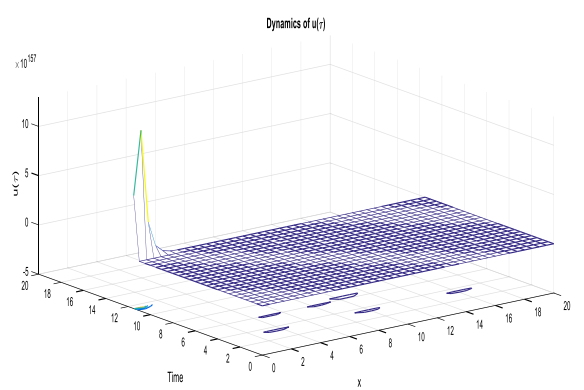

(f)

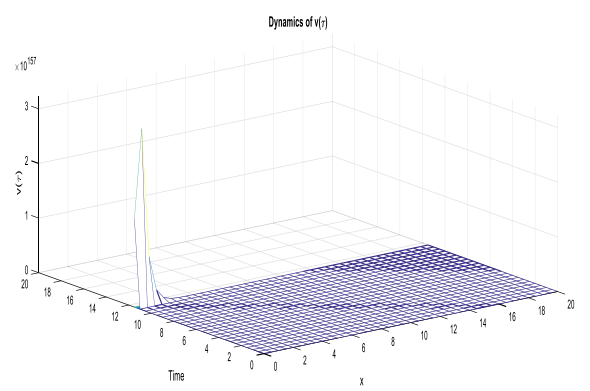

(h)

Figure $7(\mathbf{a})-(\mathbf{d})$ Numerical solutions of systems (32) and (49) using Euler method. Here, $u_{0}=1+\sin (x)$ and $v_{0}=2+\cos (x)$, with $I=5, m=1, \kappa_{1}=0.01$, and $\kappa_{2}=0.01$. The plots of solutions $v(x, \tau)$ and $u(x, \tau)$ shows divergent behavior. (e)-(h) Numerical solutions of systems (32) and (49) using Crank-Nicolson method. Here, $u_{0}=1+\sin (x)$ and $v_{0}=2+\cos (x)$, with $/=5, m=1, \kappa_{1}=0.01$, and $\kappa_{2}=0.01$ 


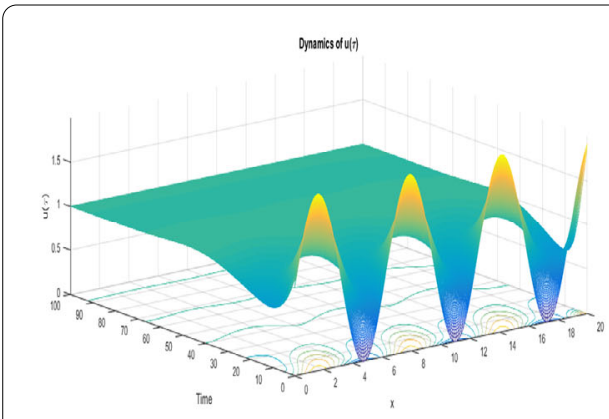

(a)

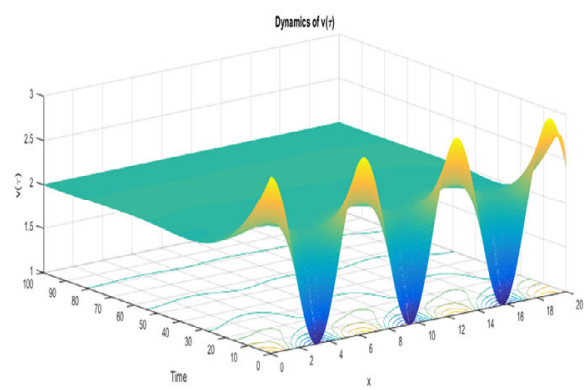

(c)

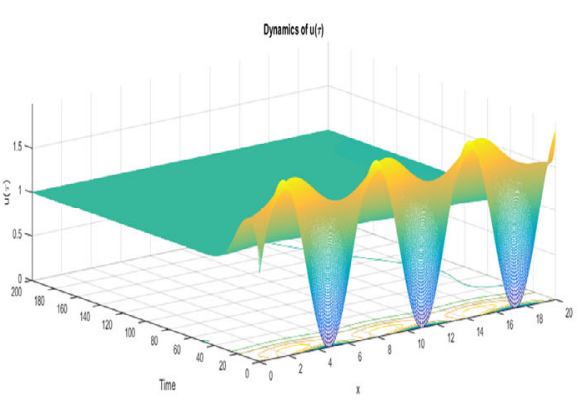

(b)

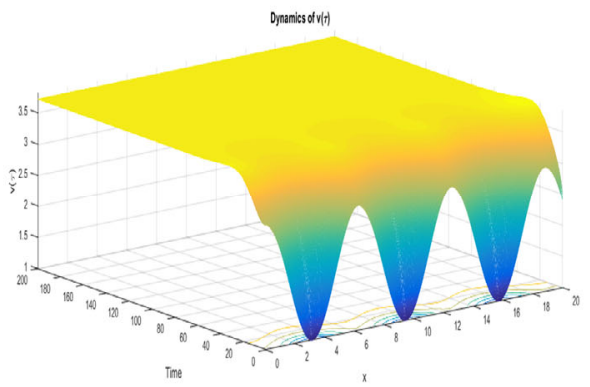

(d)

Figure 8 (a)-(d) Numerical solutions of systems (32) and (49) using the nonstandard finite difference method. Here, $u_{0}=1+\sin (x)$ and $v_{0}=2+\cos (x)$, with $/=5, m=1, \kappa_{1}=0.01$, and $\kappa_{2}=0.01$. The plots of solutions $v(x, \tau)$ and $u(x, \tau)$ show convergent behavior

$\kappa_{2}=0.01$. In all figures, the system converges to the constant steady state $(1,3.7183)$. Figures $4(\mathrm{e})-(\mathrm{h}), 5(\mathrm{e})-(\mathrm{h})$, and $6(\mathrm{e})-(\mathrm{h})$ represent the mesh plots and projected views for $u(x, \tau)$ and $v(x, \tau)$. Mesh plots and projected views of Figs. 4(e)-(h), 5(e)-(h), and 6(e)(h) indicate that the solutions $u(x, \tau)$ and $v(x, \tau)$ tend to spatially nonhomogeneous steady states. It has been observed that when we increase the value of $d \tau$ by 0.5 , the forward Euler and Crank-Nicolson schemes fail to provide stable solutions (Figs. 7(a)-(d) and 7(e)(h)), whereas the explicit nonstandard finite difference scheme provides stable solutions (Figs. 8(a)-(d)) even for $d \tau=0.1,0.5,1,10,100$.

\section{Conclusion}

In this article, we consider a nonlinear model with diffusion to review the dynamics of Lengyel-Epstein reaction model describing oscillating chemical reactions. The model demonstrates the connection between malonic acid, iodine, and chlorine dioxide. When simulating the model with the given methodology, we have recognized that the techniques are capturing to the true equilibrium nodes. The stability and uniformity of suggested techniques are also confirmed with the aid of von Neumann stability bounds and Taylor series, respectively. Besides, suggested nonstandard finite difference technique is unconditionally consistent regarding positivity property. The diagrams show that the suggested finite difference method is dynamically consistent with the performance of continuous systems. Conversely, two other well-known methods failed to preserve the positivity property and shown divergence on diverse step size $d \tau$. 
In the future, we will try to solve this system by using stochastic theory or by adding a delaying factor and then solving it using numerical techniques.

\author{
Acknowledgements \\ This project was supported by the Theoretical and Computational Science (TaCS) Center under Computational and \\ Applied Science for Smart Innovation Research Cluster (CLAS- SIC), Faculty of Science, KMUTT.
}

\title{
Funding
}

This research was funded by the Center of Excellence in Theoretical and Computational Science (TaCS-COE), KMUTT.

Availability of data and materials

Not applicable.

\section{Competing interests}

The authors declare that they have no competing interests.

\section{Authors' contributions}

All authors equally contributed to this work. All authors read and approved the manuscript.

\section{Author details}

${ }^{1}$ Faculty of Information Technology, University of Central Punjab, Lahore, Punjab, Pakistan. ${ }^{2}$ Center of Excellence in Theoretical and Computational Science (TaCS-CoE), SCL 802 Fixed Point Laboratory, Science Laboratory Building, King Mongkut's University of Technology Thonburi (KMUTT), 126 Pracha-Uthit Road, Bang Mod, Thrung Khru, Bangkok 10140, Thailand. ${ }^{3}$ Department of Mathematics, University of Malakand, Chakdara, Dir(Lower), KPK, Pakistan. ${ }^{4}$ KMUTT Fixed Point Research Laboratory, Room SCL 802 Fixed Point Laboratory, Science Laboratory Building, Department of Mathematics, Faculty of Science, King Mongkut's University of Technology Thonburi (KMUTT), 126 Pracha-Uthit Road, Bang Mod, Thrung Khru, Bangkok 10140, Thailand. ${ }^{5}$ Department of Medical Research, China Medical University Hospital, China Medical University, Taichung 40402, Taiwan. ${ }^{6}$ Department of Mathematics, Faculty of Science, King Abdulaziz University, P. O. Box 80203, Jeddah 21589, Saudi Arabia.

\section{Publisher's Note}

Springer Nature remains neutral with regard to jurisdictional claims in published maps and institutional affiliations.

Received: 1 May 2020 Accepted: 2 August 2020 Published online: 17 August 2020

\section{References}

1. Epstein, I.R., Pojman, J.A.: An Introduction to Nonlinear Chemical Dynamics. Oxford University Press, London (1998)

2. Mosekilde, E.: Topics in Nonlinear Dynamics: Applied to Physics and Economic System. World Scientific, Singapore (1996)

3. Lengyel, I., Ribai, G., Epstein, I.R.: Experimental and modeling study of oscillations in the chlorite-iodide-malonic-acid reaction. J. Am. Chem. Soc. 112, 9104-9110 (1990)

4. Din, Q., Donchev, T., Kolev, D.: Stability, bifurcation analysis and chaos control in chlorine-iodine-malonic acid reacation. MATCH Commun. Math. Comput. Chem. 79, 577-606 (2018)

5. Zafar, Z.: Fractional order Lengyel-Epstein chemical reaction model. Comput. Appl. Math. 38(3), 131 (2019)

6. Wang, T., Yang, T., Xiao, C., Sun, Z., Zhang, D., Yang, X., Weichman, W., Neumark, D.M.: Dynamical resonances in chemical reactions. Chem. Soc. Rev. 47, 6744-6763 (2018)

7. Abdelmalek, S., Bendoukha, S.: On the global asymptotic stability of solutions to a generalized Lengyel-Epstein system. Nonlinear Anal., Real World Appl. 35, 397-413 (2017)

8. Abdelmalek, S., Bendoukha, S., Rebiai, B.: On the stability and nonexistence of Turing patterns for the generalized Lengyel-Epstein model. Math. Methods Appl. Sci. 40, 6295-6305 (2017)

9. Yi, F., Wei, J., Shi, J.: Diffusion-driven instability and bifurcation in the Lengyel-Epstein system. Nonlinear Anal., Real World Appl. 9, 1038-1051 (2008)

10. Abdelmalek, S., Bendoukha, S., Kirane, M.: The global existence of solutions and Asymptotic stability of a reaction-diffusion system (2017). arXiv:1711.00976v1 [math. AP]

11. Baleanu, D., Jajarmi, A., Sajjadi, S.S., Asad, J.H.: The fractional features of a harmonic oscillator with position-dependent mass. Commun. Theor. Phys. 72, 055002 (2020)

12. Jajarmi, A., Yusuf, A., Baleanu, D., Inc, M.: A new fractional HRSV model and its optimal control: a non-singular operator approach. Physica A 547, 123860 (2020). https://doi.org/10.1016/j.physa.2019.123860

13. Baleanu, D., Jajarmi, A., Mohammadi, H., Rezapour, S.: A new study on the mathematical modelling of human lever with Caputo-Fabrizio fractional derivative. Chaos Solitons Fractals 134, 109705 (2020)

14. Jajarmi, A., Baleanu, D., Sajjadi, S.S., Asad, J.H.: A new feature of the fractional Euler-Lagrange equations for a coupled oscillator using a nonsingular operator approach. Front. Phys. 7, 196 (2019). https://doi.org/10.3389/fphy.2019.00196

15. Jajarmi, A., Arshad, S., Baleanu, D.: A new fractional modelling and control strategy for the outbreak of dengue fever. Physica A 535, 122524 (2019)

16. Baleanu, D., Jajarmi, A., Sajjadi, S.S., Mozyrska, D.: A new fractional model and optimal control of a tumor-immune surveillance with nonsingular derivative operator. Chaos 29, 083127 (2019)

17. Shiri, B., Wu, G., Baleanu, D.: Collocation methods for terminal value problems of tempered fractional differential equations. Appl. Numer. Math. (2020). https://doi.org/10.1016/j.apnum.2020.05.007 
18. Ma, C., Shiri, B., Wu, G., Baleanu, D.: New signal smoothing equations with short memory and variable order. Optik (2020). https://doi.org/10.1016/j.jijleo.2020.164507

19. Alijani, Z., Baleanu, D., Shiri, B., Wu, G.: Spline collocation methods for fuzzy fractional differential equations. Chaos Solitons Fractals (2020). https://doi.org/10.1016/j.chaos.2019.109510

20. Shiri, B., Baleanu, D.: System of fractional algebraic equations with applications. Chaos Solitons Fractals 120, 203-212 (2019)

21. Dadkhah, E., Shiri, B., Ghaffarzadeh, H., Baleanu, D.: Visco-elastic dampers in structural buildings and numerical solution with spline collocation methods. J. Appl. Math. Comput. 63, 29-57 (2020)

22. Shiri, B., Perfilieva, I., Alijani, Z.: Classical approximation for fuzzy Fredholm integral equation. Fuzzy Sets Syst. (2020). https://doi.org/10.1016/.jfs.2020.03.023

23. Zafar, Z., Rehan, K., Mushtaq, M., Rafiq, M.: Numerical treatment for nonlinear Brusselator chemical model. J. Differ. Equ. Appl. 23(3), 521-538 (2017)

24. Zafar, Z., Ahmad, M.O., Pervaiz, A.: Fourth order compact method for one dimensional homogeneous telegraph equation. Pak. J. Sci. 64(2), 144-150 (2012)

25. Zafar, Z., Ahmad, M.O., Pervaiz, A., Ahmad, N.: ZZ fourth order compact BVM for the equation of lateral heat loss. Pak. J. Eng. Appl. Sci. 11, 96-103 (2012)

26. Zafar, Z., Hussain, T., Pervaiz, A., Ahmad, M.O., Kalim, M.: A new fourth order compact difference scheme for one dimensional advection diffusion equation. Pak. J. Sci. 64(4), 359-362 (2012)

27. Mansouri, D., Abdelmalik, S., Bendoukha, S.: On the asymptotic stability of the time-fractional Lengyel-Epstein system. Comput. Math. Appl. 78(1), 1415-1430 (2019)

28. De Mottoni, P., Rothe, F.: Convergence to homogeneous equilibrium state for generalized Volterra-Lotka systems with diffusion. SIAM J. Appl. Math. 37(3), 648-663 (1979)

29. Yi, F., Wei, J., Shi, J.: Global asymptotic behavior of the Lengyel-Epstein reaction-diffusion system. Appl. Math. Lett. 22, 52-55 (2009)

30. Lou, Y., Ni, W.M.: Diffusion, self-diffusion and cross-diffusion. J. Differ. Equ. 131, 79-131 (1996)

\section{Submit your manuscript to a SpringerOpen ${ }^{\circ}$ journal and benefit from:}

- Convenient online submission

- Rigorous peer review

- Open access: articles freely available online

- High visibility within the field

- Retaining the copyright to your article

Submit your next manuscript at $\gg$ springeropen.com 